Images du travail, travail des images

6-7| 2019

Femmes au travail\&nbsp: quelles archives visuelles ?

\title{
Le métier de femmes en HLM. Archives télévisuelles des années 1960
}

The women's work in the social housing : an analysis of the 60's television archives

Laetitia Overney

\section{OpenEdition}

Journals

Édition électronique

URL : http://journals.openedition.org/itti/761

DOI : $10.4000 /$ itti. 761

Éditeur

Université de Poitiers

Référence électronique

Laetitia Overney, « Le métier de femmes en HLM. Archives télévisuelles des années 1960 », Images du travail, travail des images [En ligne], 6-7 | 2019, mis en ligne le 01 février 2019, consulté le 14 avril 2021. URL : http://journals.openedition.org/itti/761 ; DOI : https://doi.org/10.4000/itti.761

Ce document a été généré automatiquement le 14 avril 2021

Images du travail, travail des images 


\title{
Le métier de femmes en HLM.
} Archives télévisuelles des années 1960

\author{
The women's work in the social housing : an analysis of the 60's television \\ archives
}

Laetitia Overney

1 Sur l'écran de longs travellings montrent des façades d'immeuble qui n'en finissent pas. En contre-point, au sol, des petites fourmis s'activent: elles traversent les dalles de béton pour conduire les enfants à l'école. Puis elles retournent prendre l'escalier pour astiquer leur appartement moderne et confortable. Une vue aérienne oblique accentue ce gigantisme urbain : sur fond de musique jazzy, des plans-masses de plus de mille logements se succèdent sans fin. Ainsi la télévision des années 1960 nous fait découvrir un nouveau monde: les grands ensembles, les formes architecturales inédites, les femmes qui s'activent.

\section{Femmes et grands ensembles à l'image}

2 C'est un tout indissociable. À l'image, les femmes (et les enfants) sont les figures centrales des grands ensembles (Canteux, 2014, 83). En journée, quand les hommes sont au travail à l'extérieur des cités, elles se consacrent aux tâches domestiques. La bandeson insiste : des «villes sans bureau, sans usine, sans atelier, sans fumée, sans bruit, sans circulation $»^{1}$. Des cités d'un genre nouveau! Et ce sont les femmes qui les habitent, semblent nous dire les archives.

Bien au delà du travail ménager, c'est le métier de femmes en HLM qui est dans l'œil des producteurs des émissions. J'emploie cette expression en écho au titre du livre de Ménie Grégoire (Grégoire, 1964), un incroyable best-seller qui donne la parole aux femmes et pointe les changements dans les rapports entre les sexes : les ruptures nettes autant que les petits déplacements du quotidien. Un métier de femmes, au pluriel, à 
multiples facettes, parce que les rôles sont divers : travail domestique, emploi salarié, éducation, sexualité, maternité.

Quelles formes et quels sens particuliers va prendre ce métier de femmes dans les nouveaux HLM? Quel métier le logement moderne vient-il convoquer à l'écran? Quelles pratiques et quelles attitudes, quels savoirs et savoirs faire sont alors mis en vue par la télévision? Le présent article se propose d'en explorer les différentes dimensions en saisissant les archives télévisuelles dans leur contexte de production et de diffusion : la politique de construction des grands ensembles.

Historiquement, la ville est massivement associée à l'émancipation des rôles traditionnels pour les femmes: elle leur ouvre les portes du travail salarié, de l'éducation et les détache de l'emprise religieuse (Denèfle, 2004). Plus encore, les cités HLM constituent un espace de travail inédit pour les femmes. Car les nouvelles habitantes viennent tout juste de quitter un logement insalubre sans eau ni commodité et découvrent un nouvel univers spatial et des nouveaux équipements ${ }^{2}$. Aussi, leurs origines sociales sont-elles soulignées : la télévision montre de nombreuses femmes des catégories populaires. Par leur intermédiaire, les pouvoirs publics actionnent un nouveau mode de vie. Depuis l'entre-deux guerres, les femmes sont le pivot de cette mission de progrès, notamment à travers les arts ménagers qui bouleversent les manières d'habiter des classes moyennes (Martin, 1987 ; Clarke, 2005). Simultanément, un autre souci marque les politiques de construction à partir du milieu des années 1960 : enrayer la «sarcellite», ce prétendu mal des grands ensembles qui frapperait surtout les femmes de langueur, ennui insurmontable, dépression et déviances en tous genres (Tellier, 2008). Concentration de population, architecture déroutante avec des espaces géométriques réguliers, malfaçons, mauvaise insonorisation des logements, chantiers permanents, éloignement des centres villes, manque d'équipements publics et commerciaux, sont alors montrés du doigt par divers spécialistes ${ }^{3}$.

6 La télévision des trente glorieuses (Lévy, Cohen, 2007) est un précieux témoin des processus sociaux en cours, elle tend un miroir à la société alors en pleine mutation. Par l'explosion des documentaires, la question sociale et politique relative à la place des femmes se formule. Entre conformisme et émancipation, les reportages télévisés constituent un vecteur d'expression publique inédit. Ils révèlent les perceptions et les aspirations des femmes. Que disent-elles alors de leur travail, à voix haute ou à bas bruit? Qu'est-ce qui est laissé dans l'ombre ou tout juste suggéré ? Finalement, à qui parlent ces femmes à travers la télévision?

7 À, la suite de Marie-Françoise Lévy, je fais l'hypothèse que leurs différents témoignages affirment « une contestation réfléchie de [leur] "état de femmes" » (Lévy, 2007, 109). C'est leur métier de femmes en cités nouvelles qui se construit peu à peu, se dit et se montre à la télévision.

8 Pour comprendre ce qui se joue là, il faut donc renoncer à une posture critique assurée qui prétend savoir lire et décoder les images, et qui cherche la réalité derrière le masque ou l'imaginaire du réalisateur. Il faut « décevoir l'attente de qui veut lire là où il y a simplement à voir ", nous souffle Jacques Rancière $(1993,1016)$. Regarder ce qui est à l'écran, soit des femmes et des gestes de femmes, et reconnaître « la résistance du visible à se laisser lire» (Ibid.). Dès lors, il faut abandonner «l'analyse des représentations des femmes» pour remettre en cause le grand partage qu'elle introduit : d'un côté, le point de vue « savant » qui accède au secret des images et révèle le poids des représentations, et de l'autre, le point de vue des femmes, peu conscientes 
de leur condition et déterminées à agir selon une idée du féminin qu'elles ont incorporée. Les archives bouleversent profondément cet ordre des choses.

\section{Corpus et méthode}

9 Le corpus rassemblé est constitué de 26 documents conservés par l'Institut National d'Audiovisuel (liste en annexe) et diffusés entre 1959 et 1973 - période phare de construction des grands ensembles ${ }^{4}$, qui nous laisse aussi entrevoir les recompositions des rapports de genre propres aux années 1968 (Zancarini-Fournel, Artières, 2008, 435). Je n'ai retenu que les reportages et les documentaires longs (de dix minutes à une heure) dans lesquels les femmes s'expriment abondamment, par de-là le jeu journalistique des questions/réponses qui ne récolte que des paroles convenues. Ces documents reposent pour la plupart sur une enquête approfondie des réalisateurs et témoignent d'un véritable travail documentaire, source précieuse d'analyse pour le sociologue ${ }^{5}$. C'est le cas par exemple des films de 52 minutes de l'émission Les femmes...aussi, produite par Éliane Victor de 1964 à 1973.

10 Le corpus nous permet par ailleurs d'appréhender les différences d'approche entre les émissions destinées à un public féminin (Les femmes... aussi, Aujourd'hui Madame, Le magazine féminin, Hebdo femmes du JT) et les émissions de reportages « tout public » (Cinq colonnes à la Une, Hexagone, Le troisième ceil, Signes des temps, l'Avenir est à vous, JT, Des richesses et des hommes).

11 Question de méthode. J'ai retenu en priorité des visages, des corps, des gestes et des voix afin de contrer le stéréotype resté dans les mémoires (Laot, 2014) : les ménagères neurasthéniques du grand ensemble qui regardent par la fenêtre des fourmis prendre le train. Ainsi, je propose aux lecteurs des plongées dans les univers de travail de femmes singulières.

12 Pour ce faire, j'ai donc travaillé l'archive en coupant le son afin de « redresser le sens de la vue » (Peneff, 2009, 149). Dans un second temps, à l'inverse, j'ai retranscrit la bande sonore pour être au plus près des émotions et m'attacher à ces femmes en somme. J'ai scruté leur visage, revenant en arrière lorsque j'avais mal entendu, quelques mots doux de l'une d'elles susurrés à l'oreille de son enfant malade. J'ai ensuite multiplié les angles et les distances ; j'ai analysé les mouvements de caméra, la manière de filmer le corps des femmes, le rapport entre les mots prononcés et les images du travail, les effets de montage, tout en resituant ces archives dans leur contexte de production et de diffusion. Travailler mes perceptions de ces détails, c'est agrandir des traits essentiels : objets, vêtements, silence et insistance.

13 Dans cet article, j'ai choisi de concentrer le regard sur deux femmes, parce qu'elles incarnent chacune une facette du métier de femmes en HLM: le travail ménager et l'action au service de la cité. Leurs voix et leurs images feront écho à d'autres femmes filmées dans le corpus. 


\section{Le travail ménager de Micheline Hébert}

\section{1. Ce travail qu'elle ne pouvait interrompre}

\section{Séquence 1.}

"Micheline, 6 enfants, allée des Jonquilles ", Les femmes... aussi, 1ère chaîne, Claude Goretta, 24 avril 1967

Image 1. Retourner les chaises

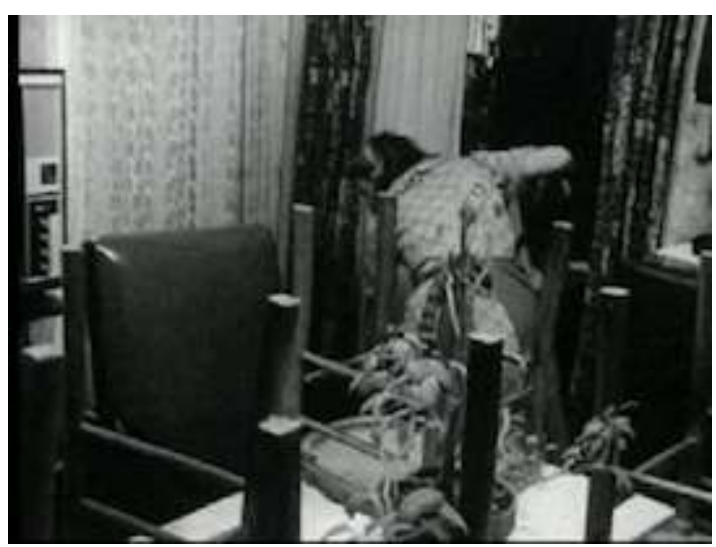

(c)Les femmes aussi - 1967

Image 2. Les jambes au travail

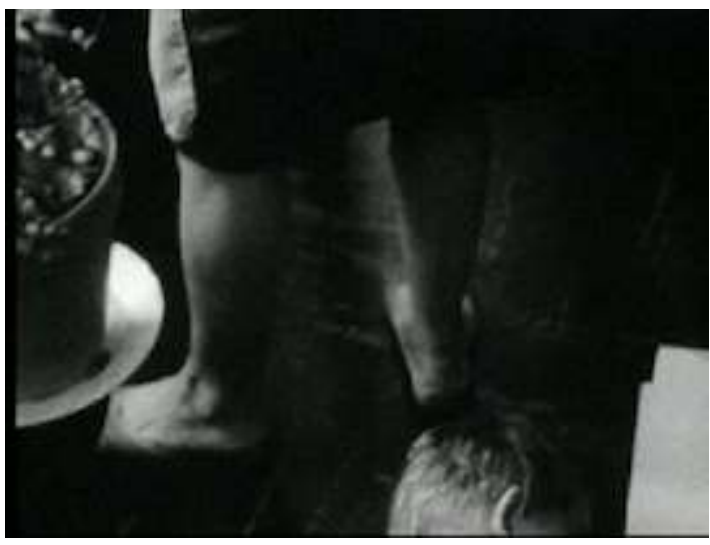

CLes femmes aussi - 1967 
Image 3. Passer la serpillère

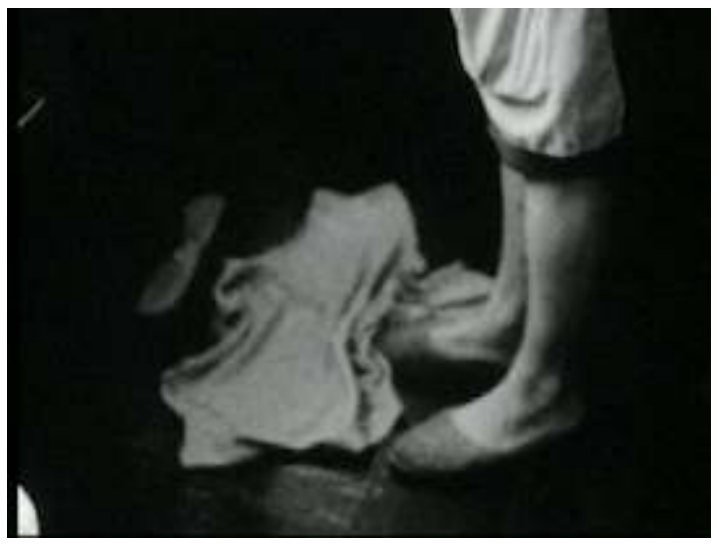

CLes femmes aussi - 1967

Image 4. Se baisser

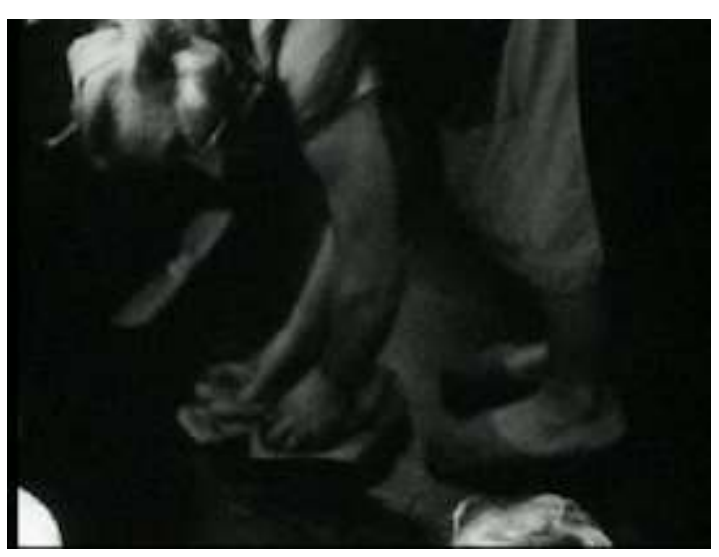

CLes femmes aussi - 1967

Image 5. Faire les recoins

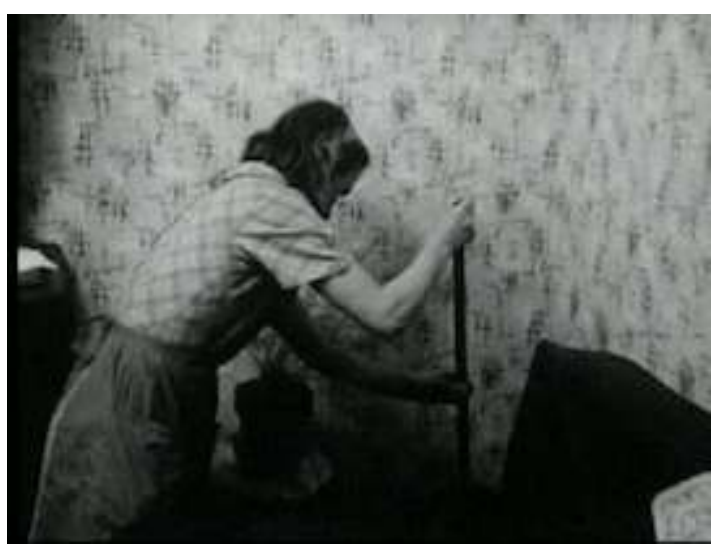

CLes femmes aussi - 1967 
Image 6. Nettoyer derrière l'oreille

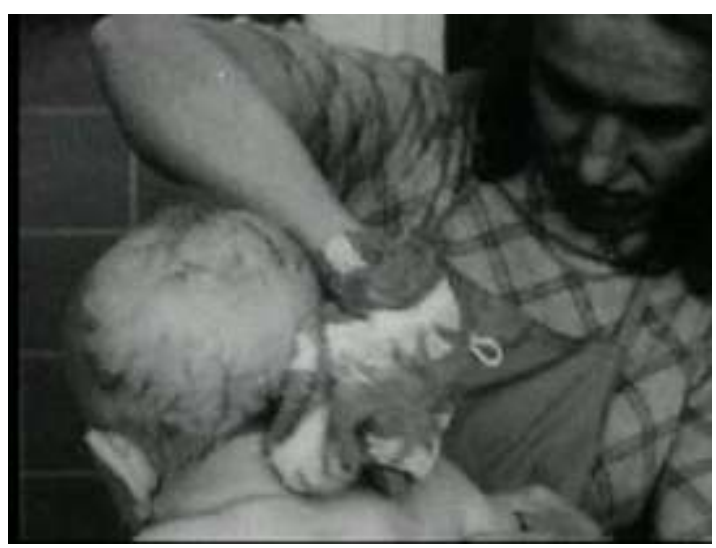

CLes femmes aussi - 1967

Image 7. Sourire à son bébé

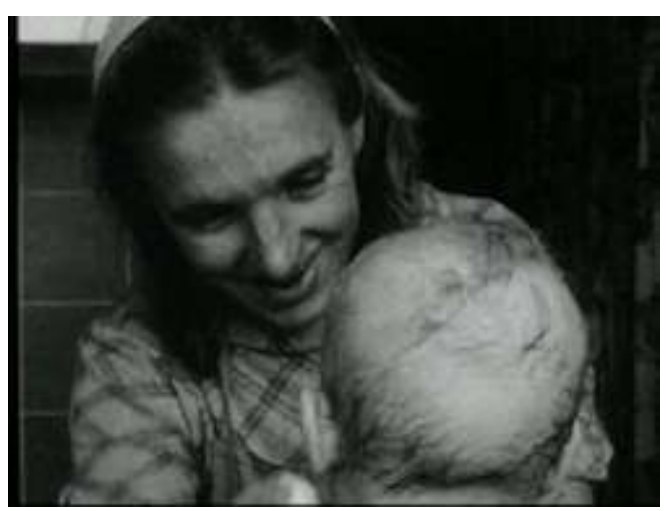

(C) Les femmes aussi - 1967

14 Les chaises sont retournées sur la table de la salle à manger. Entre le buffet, le meuble de télévision et les doubles rideaux, Micheline Hébert a juste assez de place pour passer la serpillière. La caméra filme ses hanches, drapées d'un tablier, qui ondulent à mesure qu'elle pousse le balai-brosse. Plans rapprochés sur les jambes et les piétinements de la ménagère. Elle se baisse pour passer sous les meubles, déplace le guéridon pour nettoyer un recoin. Le parquet est ciré une fois par semaine. Micheline fait son ménage tout en gardant un œil sur ses enfants qui l'entourent. Un bébé est calé sur un fauteuil en skaï, soudain il fait un renvoi, vite, il faut lui essuyer la bouche. Micheline surveille l'abcès dentaire de sa fille puis retourne à la serpillière en contournant soigneusement son fils qui dessine par terre. La caméra suit cette chorégraphie domestique pendant plus de $2 \mathrm{mn} 30$. Puis, la jeune femme passe dans la salle d'eau pour changer le bébé. La caméra s'attarde alors sur le visage de Micheline, ses doux sourires à l'enfant et son regard concentré pendant le soin. Ses mains effectuent une toilette énergique et méticuleuse. La séquence aura duré au total $6 \mathrm{mn}$. De nombreux plans sans coupe constituent ainsi le documentaire et font sentir la durée des tâches ménagères, leur superposition et leur enchaînement.

15 Micheline Hébert a 30 ans. Elle habite un immeuble HLM de Nanterre. Elle est mariée à un ouvrier, et élève six enfants, qui ont de 6 mois à 11 ans. L'émission Les femmes... aussi lui consacre un documentaire réalisé par Claude Goretta en 1967. Pendant près d'une 
heure, le réalisateur filme les moindres gestes de cette femme, tandis qu'elle se raconte à la journaliste. Le téléspectateur se trouve plongé dans le petit monde intérieur de la famille et les tourments domestiques.

16 Micheline s'est levée à $5 \mathrm{~h} 30$, comme tous les lundis et vendredis. Elle a d'abord préparé du café pour son mari. Ensuite, elle a mis bouillir sa lessiveuse à gaz pendant qu'elle faisait déjeuner ses enfants. Toilette puis habillage des bambins. Enfin, ils sont partis à l'école, Micheline a pu finir sa lessive, laver les bols et la cuisine, faire son lit et enfin nettoyer la salle à manger. Cet après-midi, elle bricolera à la maison nous dit-elle : « raccommodage, repassage, lavage, il manque toujours quelque chose, un bouton, un accroc ». Jamais quantifié, peu étudié sauf dans les écoles ménagères, soudain le travail ménager se trouve sous les feux des projecteurs.

\section{Séquence 2.}

"Micheline, 6 enfants, allée des Jonquilles ", Les femmes... aussi, 1ère chaîne, claude Goretta, 24 avril 1967

Image 8.

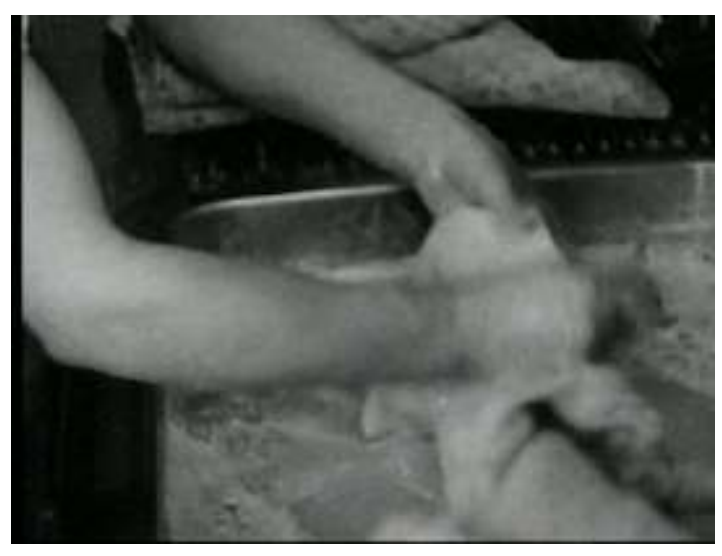

Image 9.

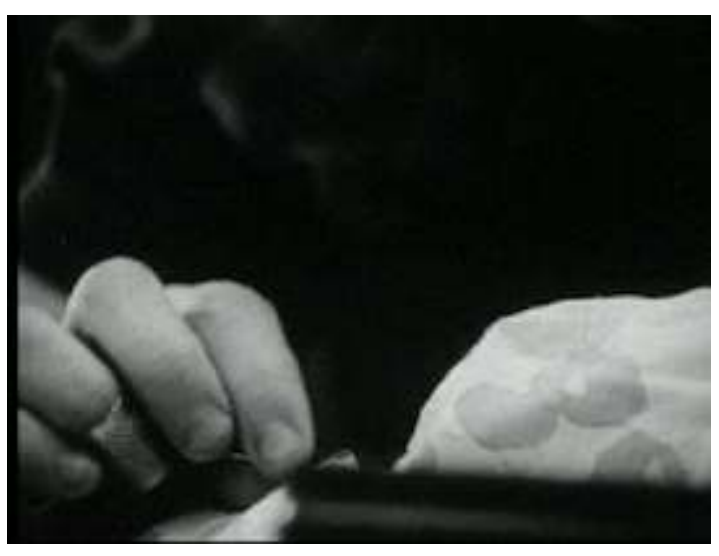


Image 10.

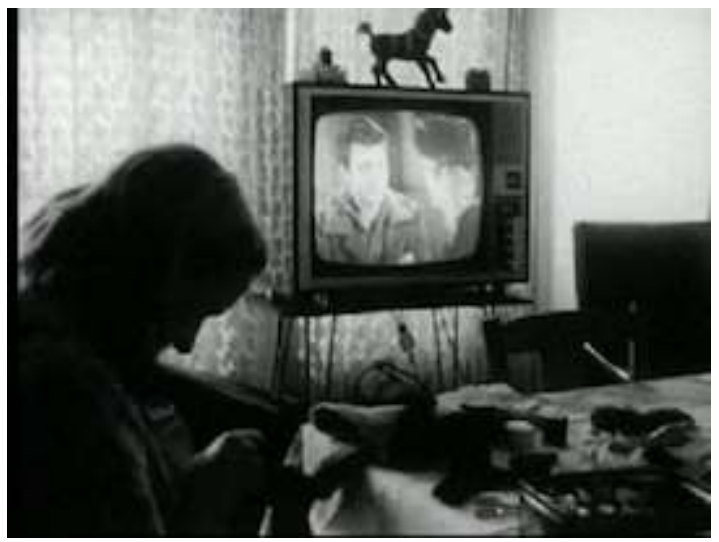

17 Micheline n'arrête pas comme on dit, même lorsqu'elle est malade. Le temps du travail domestique échappe à la mesure et se loge dans les moindres interstices (ChabaudRychter, et al, 1985). Il se caractérise par une disponibilité permanente qu'ont bien mis en relief les sociologues du care (Bessin, Gaudart, 2009). Lorsque Micheline reprise devant la télévision, elle regarde à peine l'écran. Du matin au soir doivent se succéder une série de tâches qui ne laissent aucune place ni aux distractions ni aux vacances: elle ne se voit pas « aller à la pêche avec six enfants ", et si elle sort au parc à Colombes, c'est d'abord pour les distraire. "J'ai jamais de temps devant moi » conclut-elle. Selon une étude budget-temps de 1958, une femme, qui ne travaille pas à l'extérieur passe $75 \mathrm{~h} 30$ par semaine aux tâches domestiques pour une famille de trois enfants (Girard, 1958). Micheline en a six.

\subsection{Circuler utile et compter}

\section{Séquence 3.}

«Micheline, 6 enfants, allée des Jonquilles ", Les femmes... aussi, 1ère chaîne, Claude Goretta, 24 avril 1967

Image 11. Descendre les marches avec le landau

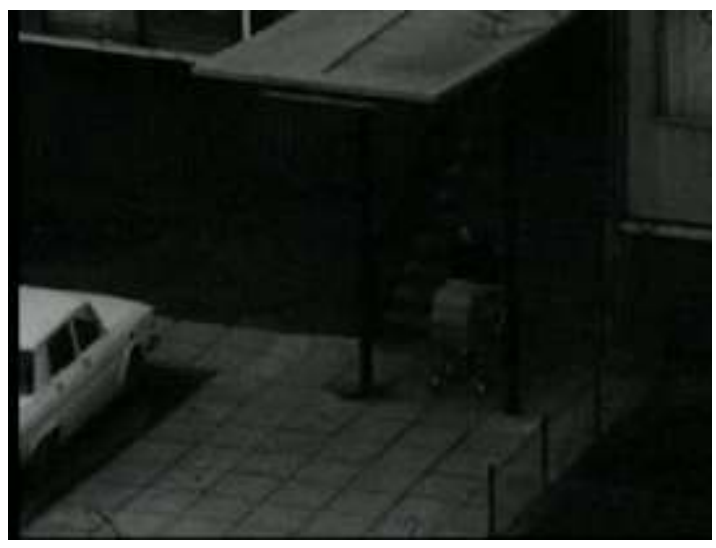

(C) Les femmes aussi - 1967 
Image 12. Aller chercher les enfants à l'école

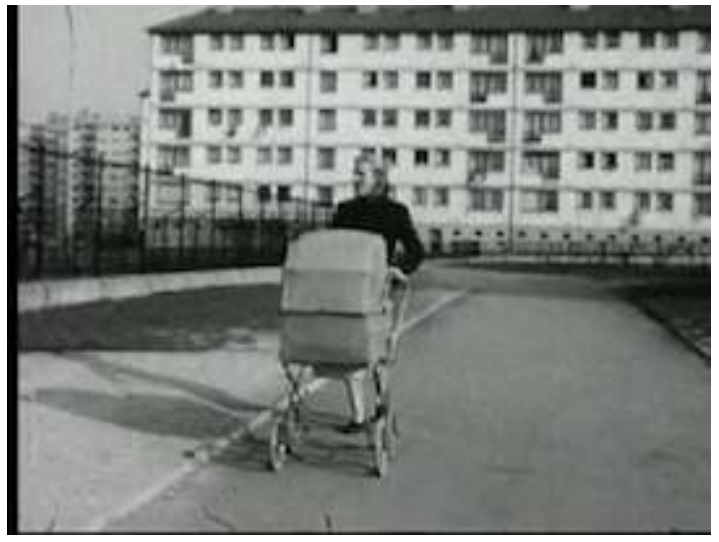

CLLes femmes aussi - 1967

Image 13. Aller aux halles

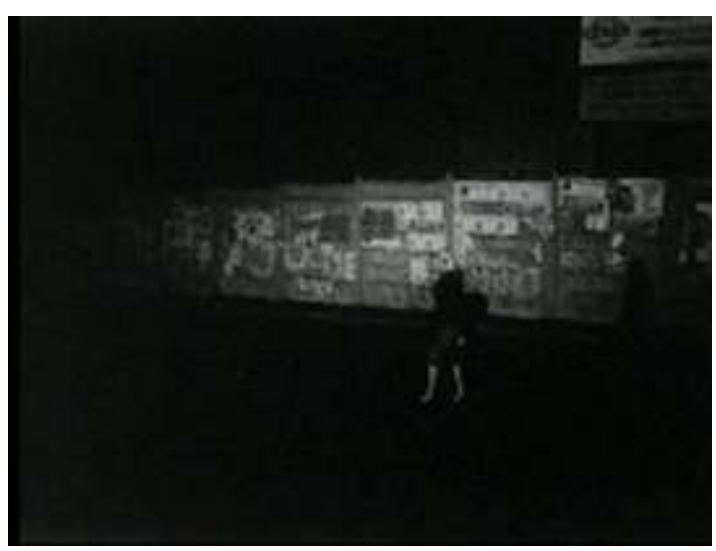

(C)Les femmes aussi- 1967

Images 14. Prendre le métro, aller aux Halles

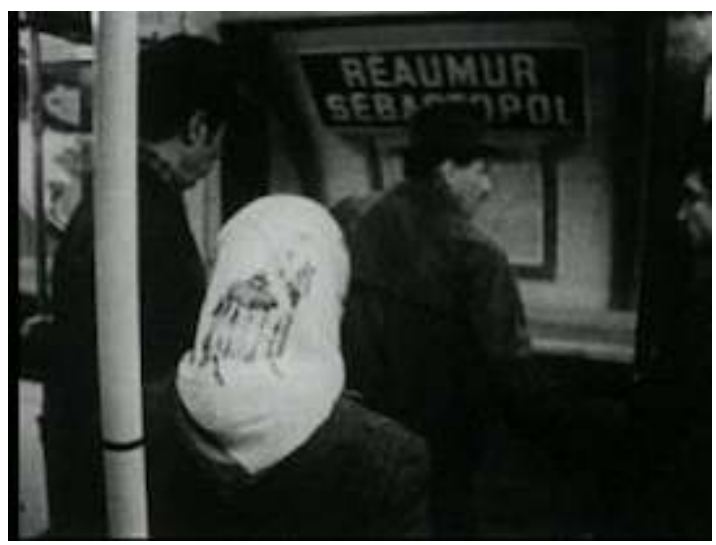

(C)Les femmes aussi- 1967 
Image 15. Aux halles

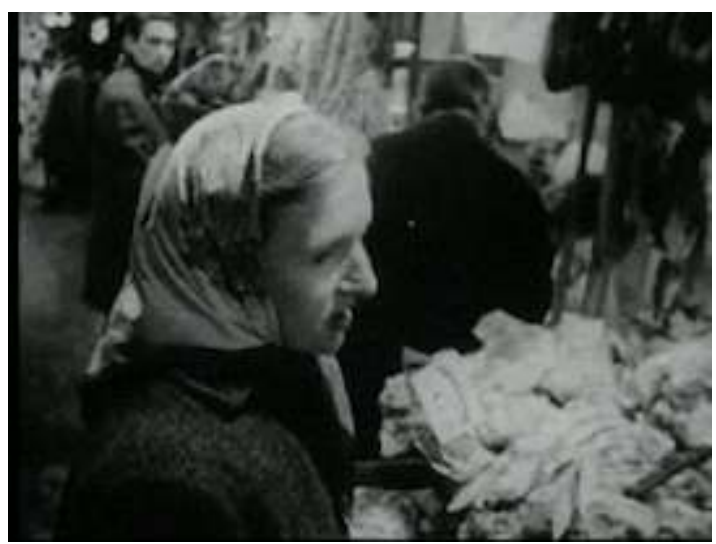

CLes femmes aussi - 1967

Le travail domestique se prolonge à l'extérieur du logis. Dès le premier plan du documentaire, Micheline marche dans l'espace public du grand ensemble. Foulard noué sur la tête - comme à chacune de ses sorties - elle descend difficilement les escaliers avec son landau, puis traverse le parking pour aller chercher ses enfants à l'école. Le film alterne scènes d'extérieur et d'intérieur, au rythme de ses nombreux déplacements. Aujourd'hui encore, les femmes circulent plus que les hommes à pied pour des motifs domestiques. Michelle Perrot nous rappelle que c'est à partir de la fin du XIXe siècle que les itinéraires féminins dans la ville se font plus canalisés que ceux des hommes (Perrot, 1980). Micheline ne flâne pas - telle la bourgeoise qui se promène le nez au vent ou lèche les vitrines - elle circule utile : elle doit filer aux Halles à Paris à 5 heures du matin pour acheter de la viande bon marché et rejoindre l'école à $11 \mathrm{~h} 30$.

\section{Séquence 4.}

"Micheline, 6 enfants, allée des Jonquilles ", Les femmes... aussi, 1ère chaîne, Claude Goretta, 24 avril 1967

Image 16. Le porte monnaie en main

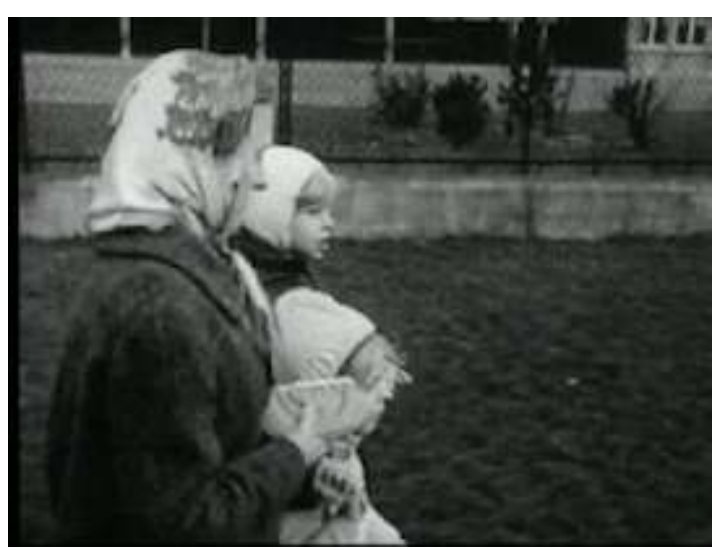

(C) Les femmes aussi - 1967 
Image 17. Le mari rentre du travail

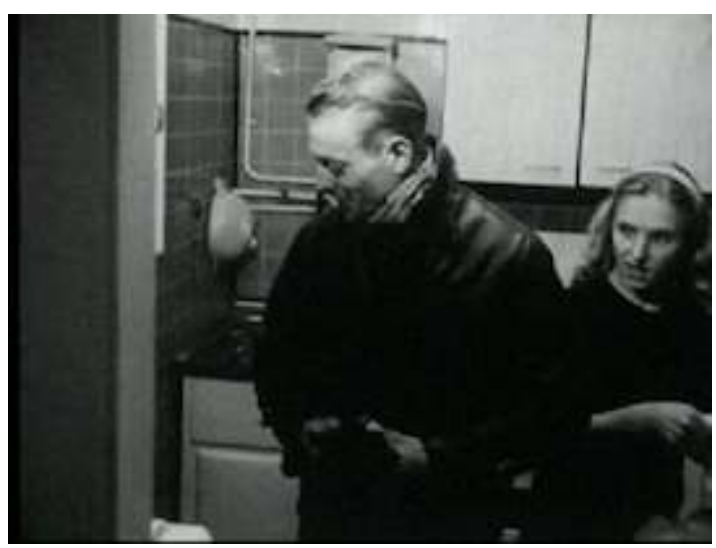

CLLes femmes aussi- 1967

Image 18. La remise de la paie

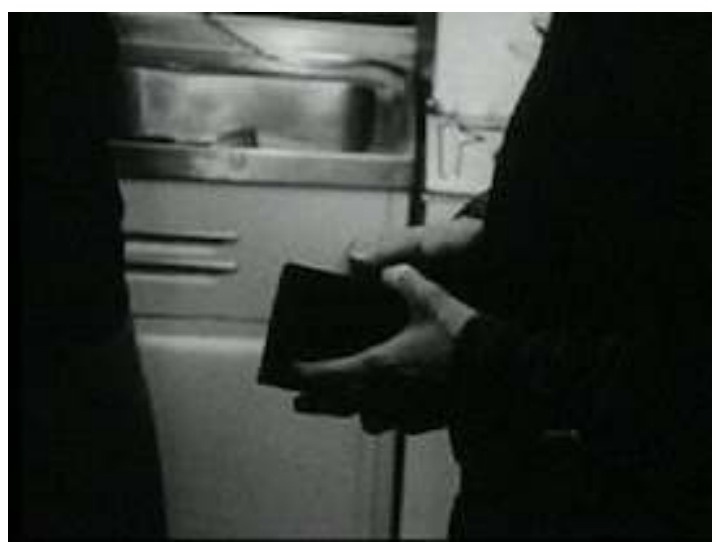

(c)Les femmes aussi - 1967

19 Micheline sort le porte-monnaie en main. Le budget, «ce sont les affaires de ma femme » explique Monsieur Hébert avec un sourire gêné. À l'image, une scène rituelle, un geste séculier dans les familles populaires : la remise de la paie de la semaine par le mari à sa femme. Part importante du métier de femme en HLM, la responsabilité de l'économie domestique apparaît plus comme une charge complexe qu'un privilège. Le documentaire nous montre une femme qui n'arrête pas de compter: les allocations familiales qui couvrent le loyer, le gaz et les traites du meuble à crédit, la paie du mari pour « la nourriture, les vêtements des enfants, leur cantine, les produits d'entretien »; elle évalue l'« écart terrible » de 300 à 400 francs par kilo de viande entre le prix des Halles et celui des commerces du grand ensemble, ou encore l'économie réalisée en ramassant des boutons par terre ou en n'allant jamais chez le coiffeur. "Se défendre " aux Halles (marchander) signifie encore de faire des économies ${ }^{6}$.

Micheline dégage un salaire d'appoint qui l'aide à payer le patronage pour ses enfants : elle lave à domicile le linge d'autres familles. Elle cherche à équilibrer un budget toujours précaire en disciplinant la consommation familiale par exemple lorsqu'elle refuse à son fils l'achat de limonade. Le couple évite les crédits « pour pas tirer quand même ", pour ne pas (trop) se serrer la ceinture jusqu'à l'étranglement. Femme modeste, Micheline ne peut pas se permettre d'être négligente. 
«Une famille [modeste] qui donne l'impression de tenir le coup » résume Monsieur Marie, «directeur du Groupe Scolaire des Pâquerettes » où sont scolarisés les enfants Hubert. L'expertise et la caution morale de l'instituteur sont données dès les premières minutes du documentaire : la famille se tient bien parce que Micheline tient bien son ménage.

\subsection{Apprendre le métier à d'autres : les conseillères ménagères}

Parce que certaines femmes s'en sortent moins bien que Micheline, il faudra leur apprendre à habiter un logement moderne, tenir une famille, gagner du temps, rationaliser les tâches, découvrir les nouvelles techniques. La télévision pourrait-elle servir cette cause? Sans conteste, une "morale domestique de l'épouse au foyer " (Lévy, 1995, 180) agit pour acculturer le public féminin à leur nouveau métier de femmes en HLM. Deux types d'émissions s'y attellent: les émissions féminines de conseils et celles qui mettent en scène une nouvelle profession: les fameuses conseillères ménagères.

\section{Séquence 5.}

" La femme chez elle ", Le magazine féminin, 27 février 1959, 1ère chaîne

Image 19. Rubrique Conseil Pratique

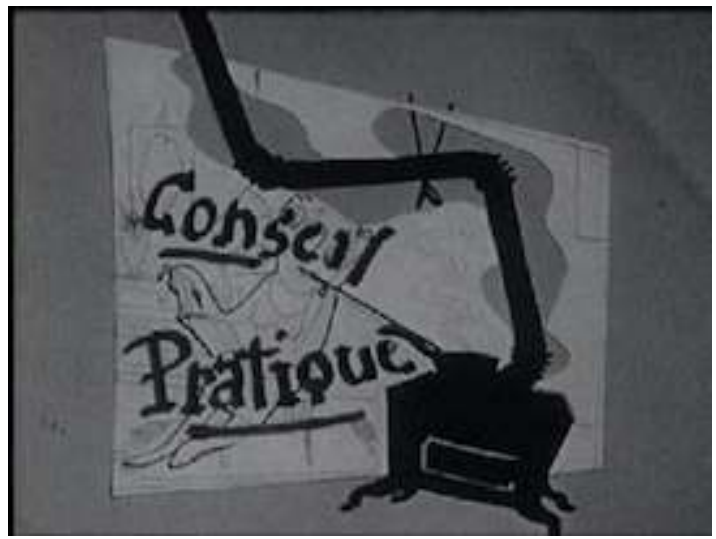

(C) Le Magazine féminin - 1959

Image 20. Techniques de balayage

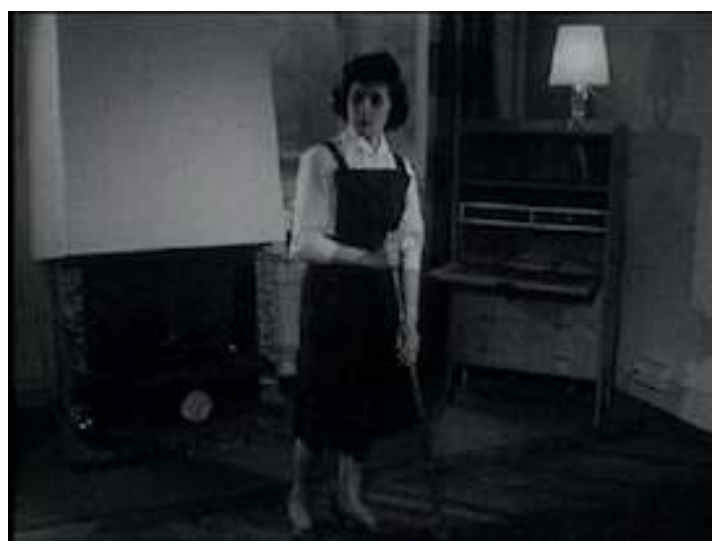

(C)Le Magazine féminin - 1959 
Image 21. Les avantages de la table roulante

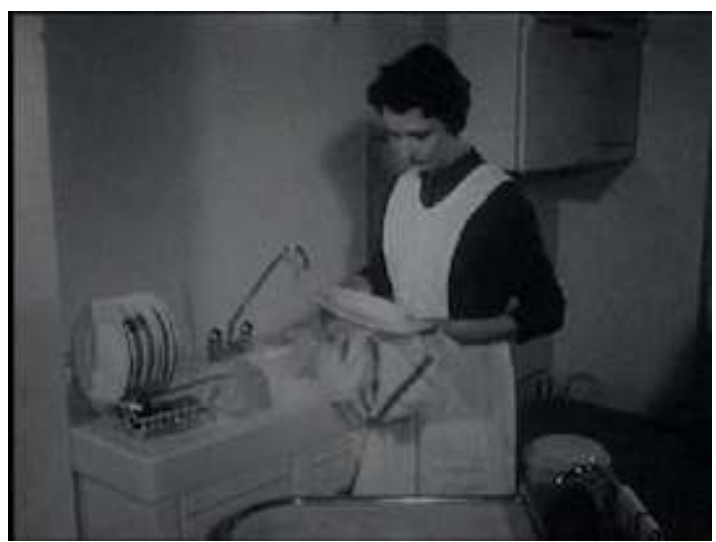

(CLe Magazine Féminin - 1959

Les programmes destinés aux femmes ${ }^{7}$ assument un rôle pédagogique et mettent en valeur la rationalisation du travail ménager. En effet, chaque programme délivre des « conseils pratiques »- c'est d'ailleurs le nom d'une rubrique du Magazine féminin : de véritables leçons sur les techniques de balayage, de coupe/couture, démonstration de l'intérêt de la table roulante, etc. Les gestes techniques sont décortiqués à l'image, un principe routinier de l'enseignement ménager (Brayet, 2010), dont la télévision relaie les fondamentaux ${ }^{8}$. L'image prend alors une valeur normative: la bonne manière de faire, le geste juste. Aguerrie aux meilleures techniques, la femme gère en experte les multiples travaux ménagers. Elle est montrée comme « un chef d'entreprise qui se doit de penser aux rendements ${ }^{9}$. Elle profite de la mécanisation des foyers et d'une esthétique ménagère (cuisines modernes, électro-ménager, décoration) qui annoncent la société de consommation. Les ustensiles modernes du métier de femmes en HLM sont partout sur les écrans: meuble en formica, vaisselle en pyrex, chauffe-eau à gaz, gazinière, frigidaire, boîte à pain métallique. Des femmes témoignent : le confort du HLM facilite le travail ménager : Ernestine, trois enfants, anciennement logée dans un cabanon de jardin, explique comment aujourd'hui il lui faut trente minutes pour la grande toilette quand il lui fallait plus d'une heure auparavant ${ }^{10}$.

Cette puissante mission éducative des HLM va faire appel aux caisses d'allocations familiales et à EDF qui recruteront à cet effet des conseillères ménagères et des monitrices d'enseignement ménager familial (Faure, 1992). N'est-ce pas la meilleure façon de lier les prestations sociales aux bons usages de celles-ci? Lorsqu'on vient d'une courée du 13e arrondissement de Paris, savoir habiter un HLM ne va pas de soi. De nouveaux problèmes ménagers surgissent.

\section{Séquence 6.}

"Conseillère ménagère ", Anne-Marie Ullmann, L'avenir est à vous, 1ère chaîne 16 mars 1964 
Image 22. Conseils d'aménagement par une conseillère ménagère

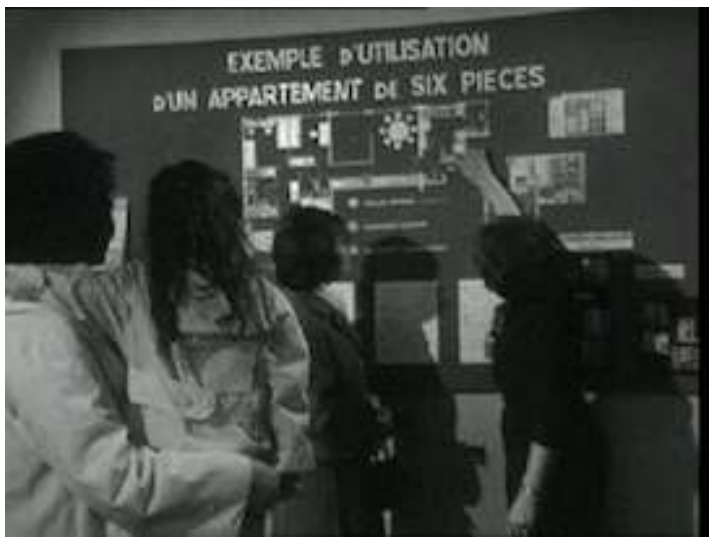

CL'avenir est à vous - 1964

Image 23. Gros plan sur la cuisine

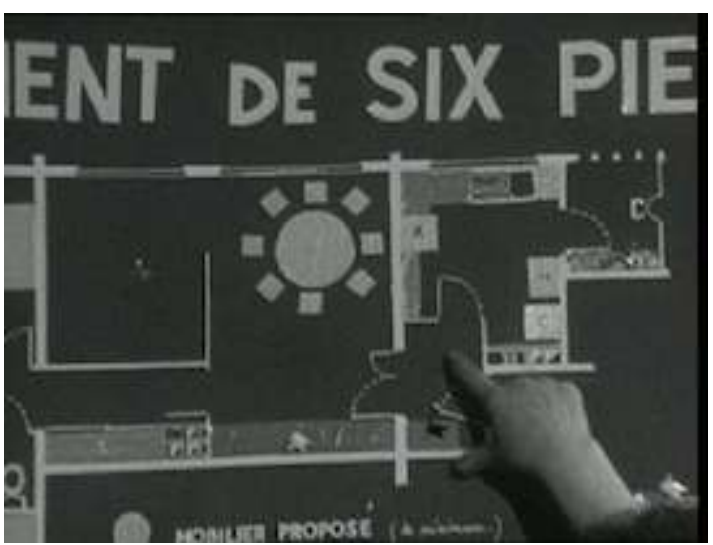

CL'avenir est à vous - 1964

\section{Séquence 7.}

"Les arts ménagers au service du foyer", Des richesses et des hommes, 27 novembre 1961, 1ère chaîne

Image 24. Entretien avec une conseillère ménagère

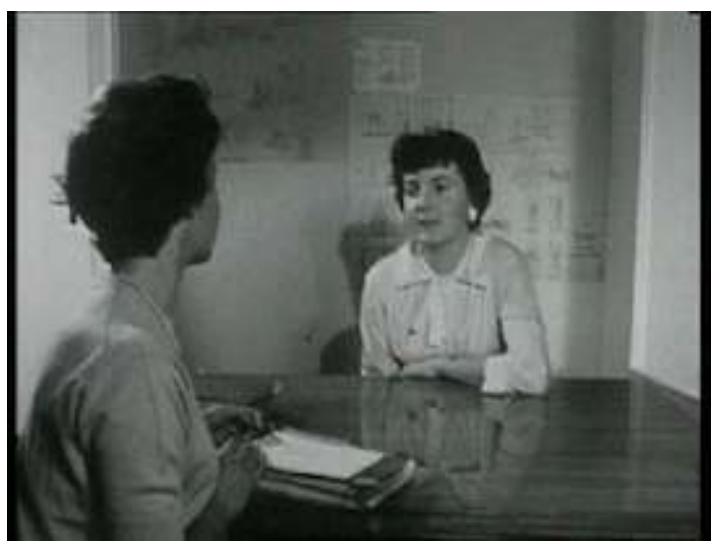

(C) Des richesses et des hommes - 1961 
Image 25. Entretien avec une conseillère ménagère 2

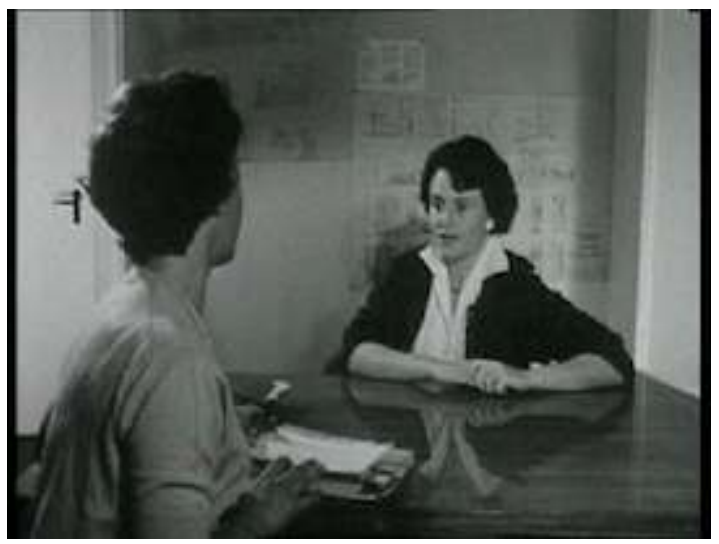

CDes richesses et des hommes - 1961

Lors de ces émissions, les rapports dissymétriques entre les conseillères et les femmes sautent aux yeux : la conseillère accentue sa position d'enseignement. Elle reçoit assise à son bureau, donne une leçon de cuisine, des conseils d'aménagement. Elle explique aux téléspectateurs l'importance du contrôle du budget en martelant : «les femmes se mettent des dettes sur le dos, et c'est très ennuyeux. C'est là que commence l'angoisse et on a vu des catastrophes ", et de donner quelques directives : hiérarchiser les achats de meubles, s'exercer au petit bricolage, connaître les commerçants les moins chers.

\section{Séquence 8.}

"Visite d'un appartement témoin HLM à Bron Parilly ", Actualités Rhône Alpes, 2 décembre 1960

Image 26. D'astucieux rangements

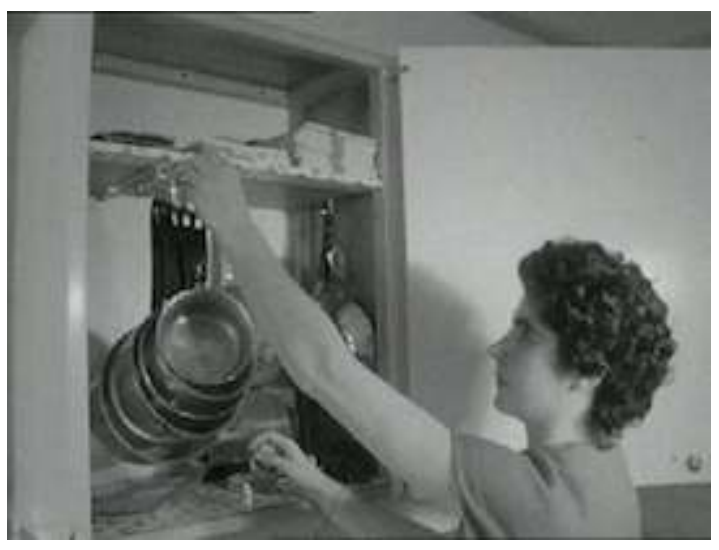

C.JT Rhône-Alpes - 1960 
Image 27. Du linge soigneusement plié

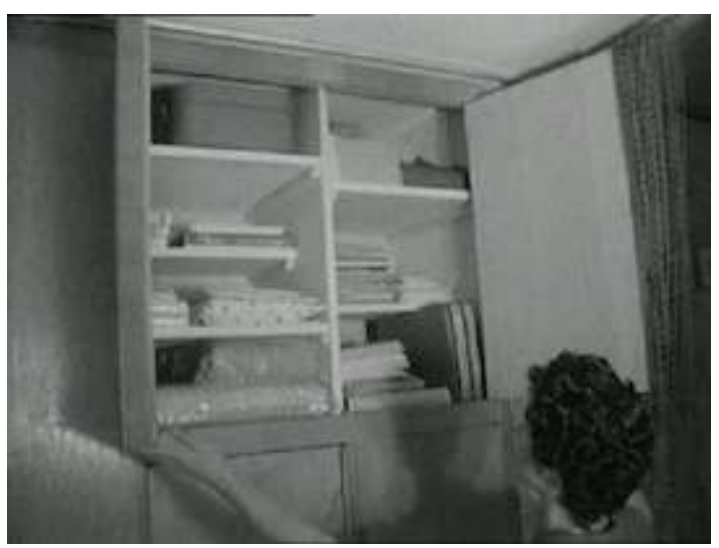

C.JT Rhône-Alpes - 1960

Une autre présente un appartement-témoin pendant $2 \mathrm{mn} 30$ en s'arrêtant sur les vastes placards rangés de manière rationnelle, là ce linge parfaitement plié, ici des astucieux rangements des ustensiles de cuisine.

\subsection{Forces expressives : corps, émotions, paroles}

Revenons un instant à Micheline. Celle-ci se débat avec l'ordre disciplinaire. Dans les documentaires qui donnent longuement la parole aux femmes, sont mises en vue des ménagères issues des milieux populaires qui pensent leur métier de femmes en HLM.

Le réalisateur filme de près le corps de Micheline au travail. Le corps parle. Ce que l'on retient des $52 \mathrm{mn}$ passées avec elle, ce sont des va-et-vient constants entre son corps et son visage, la force de ses expressions, la puissance des émotions qui y passent: la concentration (sourcils froncés), la réflexion (elle fait la moue), ses doux sourires au bébé, la joie et la tendresse qui éclatent sur son visage quand elle danse et chante avec ses enfants sur Berthe Sylva - "Les yeux des mamans sont des étoiles ». Dans cette scène émotionnelle finale, Micheline est filmée de si près que l'on voit la transparence de ses yeux. Le téléspectateur plonge dans son regard, à la rencontre d'un être singulier.

\section{Séquence 9.}

"Micheline, 6 enfants, allée des Jonquilles ", Les femmes... aussi, 1ère chaîne, Claude Goretta, 24 avril 1967 
Image 28. Concentrée

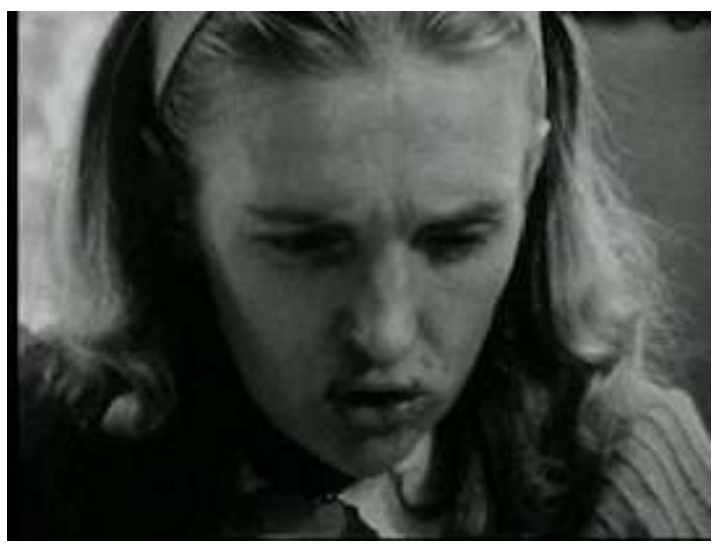

CLes femmes aussi - 1967

Image 29. La moue

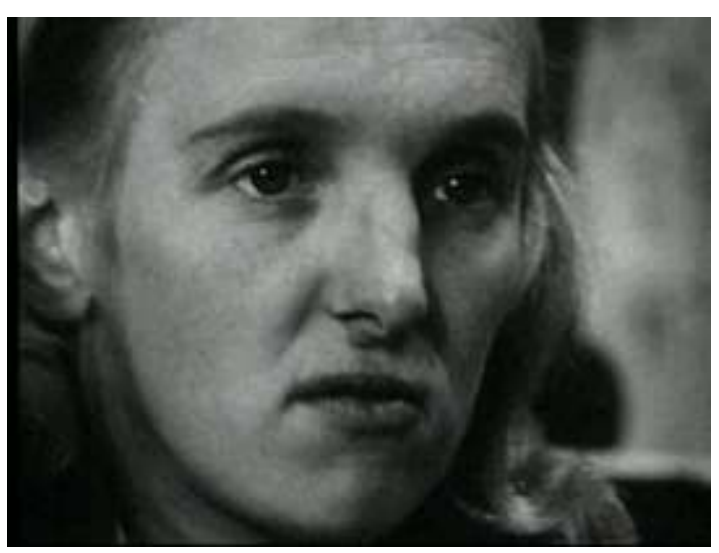

CLes femmes aussi - 1967

Image 30. Réflexion

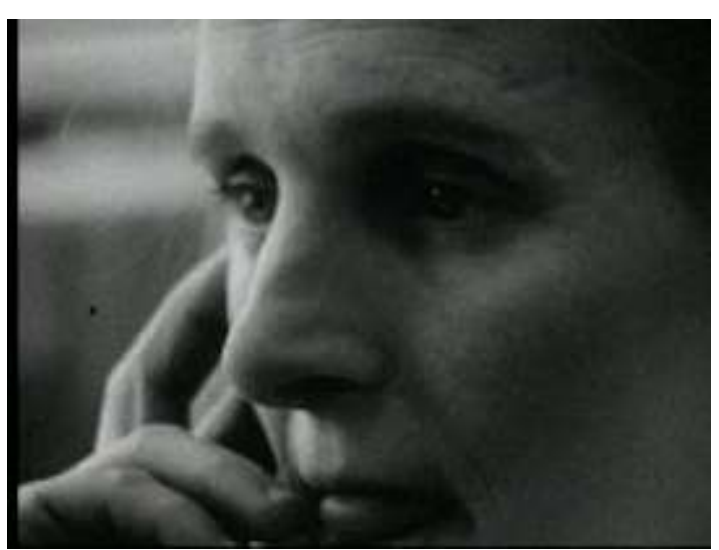

(C) Les femmes aussi - 1967 
Image 31. Tendresse

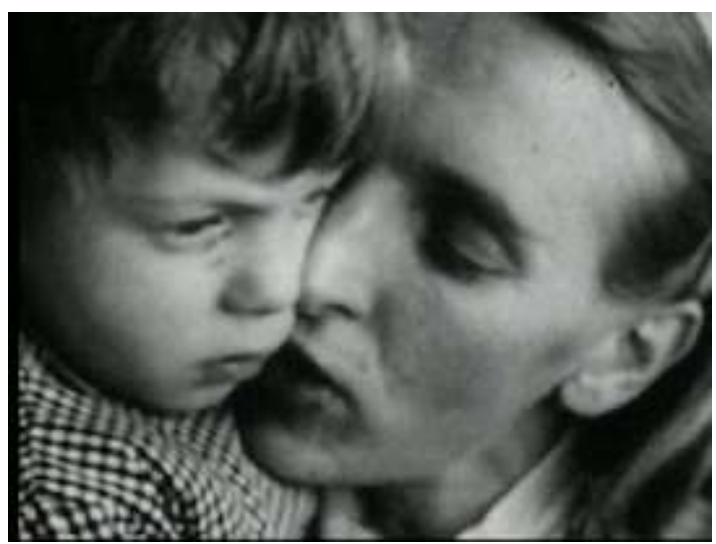

(C) Les femmes aussi - 1967

Image 32. Les yeux des mamans sont des étoiles

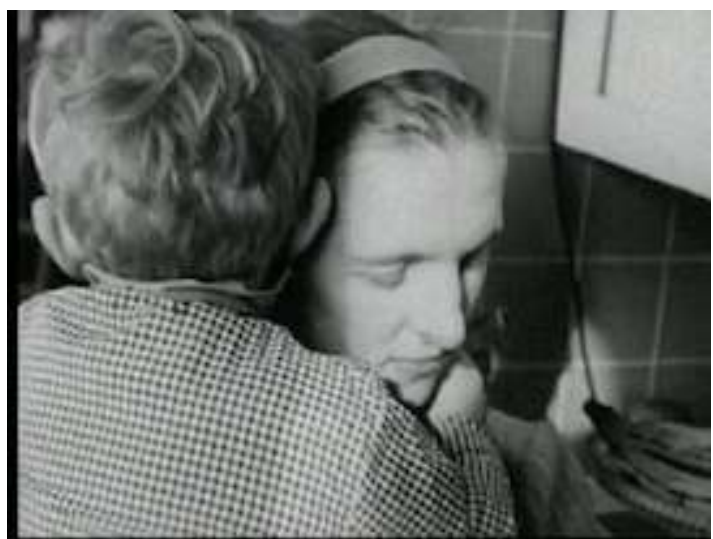

CLes femmes aussi- 1967 moderne qui comprend et agit avec son temps, les années 1960. Tout d'abord, elle se montre attentive à la scolarité de ses enfants. Ensuite, elle a un sens de la psychologie, elle brosse le portrait de chacun de ses enfants et en déduit les attitudes à adopter : l'aîné est " très émotif, on peut rien lui dire qu'il se met à pleurer ", l'une des filles est " très très calme. [Elle] évite de la brusquer ", quand Jean-Luc est présenté comme " un petit voyou [qui] a très bon caractère. [Elle] peut lui mettre une fessée, dix minutes après il viendra [l']embrasser ». Marie-José Chombart de Lauwe a bien montré comment dans les années 1960 les femmes des milieux ouvriers se montrent plus attachées que les hommes à une meilleure compréhension des enfants, un signe de progrès par rapport aux générations précédentes (Chombart de Lauwe, 1964) ${ }^{11}$. De plus, Micheline s'est forgée des connaissances médicales : sa fille $a$ un abcès, en attendant la visite chez le dentiste, elle lui fait faire des bains de bouche. Elle raconte combien elle est passionnée par les émissions médicales. Les images télévisuelles apportent du savoir dans les foyers populaires. Enfin, elle est attentive à son environnement. Dans cette cité 
moderne, elle craint les mauvaises fréquentations et les bêtises des jeunes laissés sans surveillance : carreaux cassés, œil crevé, accident de vélo. Alors, Micheline a inscrit les siens aux activités du patronage.

31 Pourtant, en cette année 1967, Micheline est déjà en décalage avec les questions qui agitent les femmes sur le travail, la maternité, la sexualité. L'entretien est conduit par l'écrivaine Françoise Mallet-Joris qui ouvre la séquence d'un : «nos deux formes de vie ne sont pas comparables »! Comme pour souligner qu'entre cette mère de six enfants et l'écrivaine, un gouffre s'ouvre.

Mais Micheline en a cure. Elle ne parle pas tant à l'écrivaine qu'aux femmes qui la regardent. Son expérience parle fort. Elle tend le miroir et interpelle celles qui partagent cette même condition. Lorsqu'elle ponctue ses propos de "vous savez... », elle s'adresse à celles qui savent la dureté de boucler les fins de mois, le travail que représentent six enfants, de les laver deux par deux tous les soirs. Son histoire vaut pour d'autres femmes.

33 Autre moment crucial : des mots couverts, des silences, pour évoquer la contraception : «mes enfants, je les mettrai à la page (...) parce que c'est beaucoup trop de travail d'avoir tant d'enfants ». Parler de contraception est interdit dans la programmation télévisuelle avant les lois Neuwirth et Weil (Sohn, 1997, 287). De sorte que s'invente tout un langage dans de nombreux documentaires, des énoncés qui ne disent pas l'interdit mais tourne autour. Si le métier de femmes en HLM est trop lourd, c'est aussi parce que l'appartement, obtenu après tant d'années d'attente, n'est déjà plus à la taille de la famille.

Micheline n'est pas dupe: sa condition vient de son manque d'instruction. L'école ménagère, « ce n'est pas l'école » lance-t-elle sans détour à l'intervieweuse. Quelle force du propos si on le prend au pied de la lettre! Il déjoue le message de l'enseignement ménager martelé depuis les années 1930: apprendre aux femmes à supporter bravement la pauvreté (Lebeaume, 2014, 46). Micheline conteste, réplique : l'école ménagère ne donne ni l'instruction, ni la liberté ${ }^{12}$.

En somme, les documentaires des années 1960 ne mettent pas publiquement en débat les changements qui s'opèrent dans la vie intime, familiale et sociale des femmes. Mais en s'exprimant ainsi à l'écran, Micheline et les autres parlent au public. Ce faisant, elles encouragent et soutiennent les débats intérieurs des femmes qui les écoutent dans leur HLM.

\section{Au service de la cité}

Changement d'échelle. Intéressons-nous maintenant à d'autres rôles sociaux. En contre-point au travail domestique, les femmes prennent une place d'animatrice de la vie sociale et politique des cités. Quelles formes va prendre à l'écran l'implication des femmes dans le quartier et dans la communauté politique ? Les archives dévoilent cette autre dimension. Notamment dans la série Les femmes... aussi, en octobre 1972, avec le documentaire "Une femme dans la cité ", soit le portrait de Marie, qui habite un grand ensemble de Marseille où elle tient une droguerie ${ }^{13}$. Elle est filmée dans la cité : le téléspectateur la découvre qui arpente les espaces publics du quartier et les cages d'escalier. Elle passe successivement de son commerce, à la salle de réunion de la maison de quartier, ou encore au logement de sa mère âgée, si bien qu'il faut attendre 
la quarantième minute pour la voir filmée dans son intérieur, entourée de son mari et de son fils. Le contraste avec la vie domestique de Micheline est saisissant. Une vie d'intérieur versus une vie à l'extérieur. Ou plus exactement, le téléspectateur découvre comment le grand ensemble devient le support d'une nouvelle vie publique pour les femmes. Des villes qui sortent de terre, des habitants qui viennent de différents horizons géographiques et sociaux, des équipements collectifs, autant de promesses pour de nouvelles sociabilités.

\subsection{Tenir commerce, délier les langues}

\section{Séquence 10.}

"Une femme dans la cité ", Maurice Dugowson, Les femmes... aussi, 1ère chaîne, 9 octobre 1967

Image 33. Tenir commerce

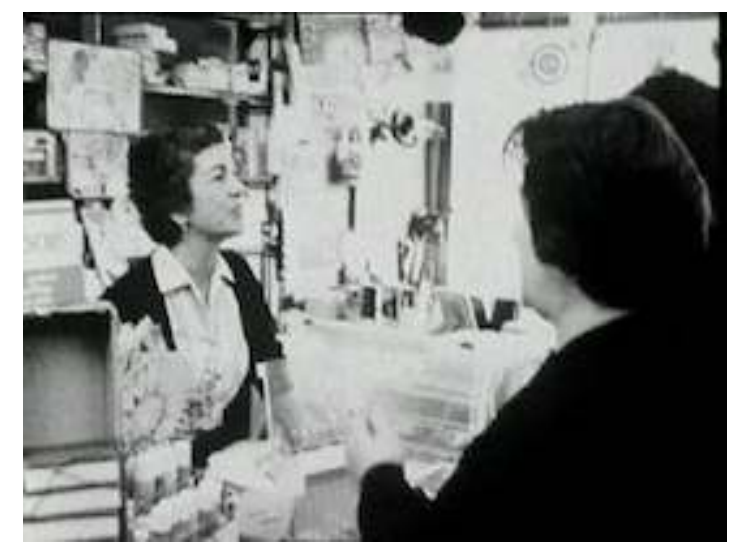

(C) Les femmes aussi- 1972

Image 34. Délier les langues

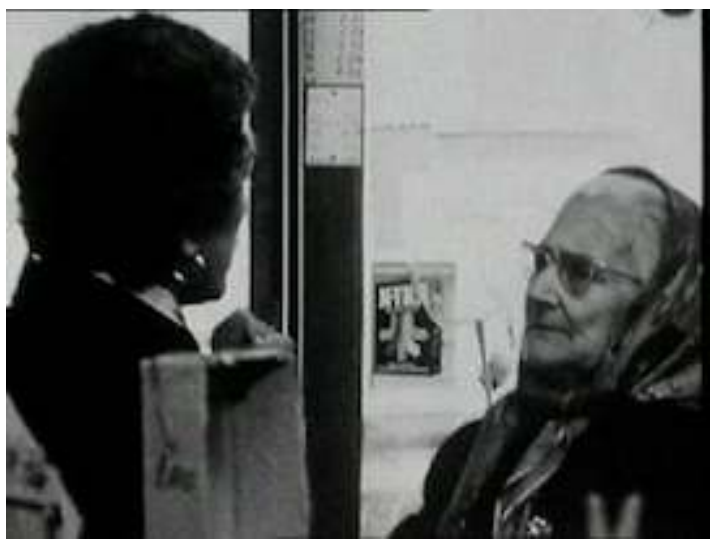

(C)Les femmes aussi - 1972

Dans une première scène, Marie bavarde à la caisse de sa boutique, elle prend des nouvelles : auprès d'une grand-mère, comment va le petit-fils resté en Espagne ? Le fils d'un client a-t-il trouvé du travail ? Est-ce que les habitants de la tour n'ont pas trop souffert du vent hier? Marie délie les langues. Le regard attentif et concentré, elle recueille les plaintes. 
On ne vient pas seulement acheter dans son magasin. Un client l'interpelle : il veut changer d'appartement. Marie lui conseille de voir l'assistante sociale en insistant sur l'exiguïté de son logement. Un autre critique le bailleur HLM qui expulse les locataires quand ils ne paient pas le loyer. Marie le rassure en faisant valoir le soutien de l'amicale des locataires. La commerçante est historiquement une femme de la rue, une femme au cœur de l'espace public et des relations sociales, une femme de contacts. La drogueriebazar est un espace mixte à plus d'un titre : il est aussi bien fréquenté par les hommes et que par les femmes, les plus modestes y achètent des produits de base, ceux qui sont plus à l'aise des appareils électro-ménagers; enfin, toutes les générations y trouvent leur bonheur. La boutique fait tenir les liens et les sociabilités dans la ville. Marie incarne une figure de vigilance vis-à-vis du quartier (Overney, 2014): une écoute immédiate des problèmes et de multiples attentions à la vie quotidienne des habitants.

Aux côtés de la commerçante, une autre profession se situe au cœur de la cité: l'infirmière à domicile. C'est le cas de Mme Robert, interviewée en 1964, qui vit et travaille à Massy-Antony ${ }^{14}$. Les habitantes qui souffrent de "dépressions nerveuses " s'ouvrent à elle : elles pleurent toute la journée, « ne voient rien que des bâtiments, des grues partout, pas de magasin ». Certaines déménagent, d'autres font des cures de sommeil. Huit ans plus tard, la télévision retrouve l'infirmière : elle a créé «le club mamans " qui propose des cours, des activités et des conférences aux mères de famille ${ }^{15}$.

\section{Séquence 11.}

"Ils ont fait cette ville", Jean-Pierre Chartier, Janick Arbois, Signes des temps, 2ème chaîne, 24 juillet 1972

Image 35. Sortie en car

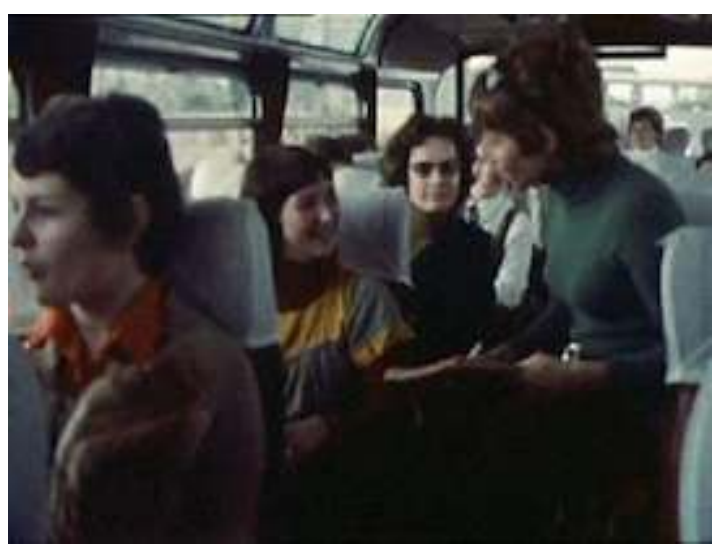

CSignes des temps - 1972 
Image 36. Rencontre au marché

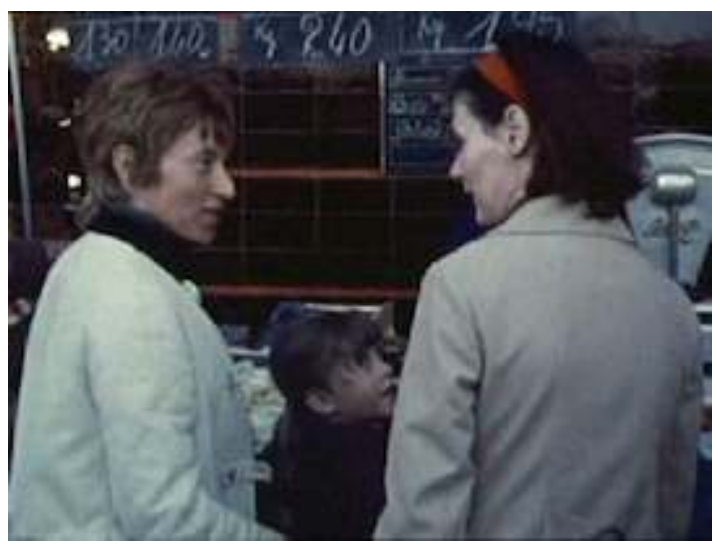

(c) Signes des temps - 1972

40 Figure du quartier, lorsqu'elle fait ses courses au centre commercial, elle est arrêtée à chaque pas par des habitants qui l'interpellent. Vie privée et vie publique se mêlent. La disponibilité du care domestique se rejoue dans le rôle public au sein des cités HLM.

\subsection{Un accent de travail social communautaire}

41 Marie agit dans sa boutique, et elle est aussi engagée comme bénévole à la maison de quartier où elle organise tous les jeudis des activités pour les enfants. Son métier de femmes en HLM, elle le conçoit là comme une mission de prévention : " on fabrique les voyous vous savez. On leur donne tous les éléments pour qu'ils le deviennent. Il faut pouvoir leur donner autre chose, les intéresser ».

Surtout, pour Marie, se mettre au service de la cité, c'est organiser une parole collective des habitants. Pour ce faire, il lui faut «faire parler » les femmes, celles qu'elle ne voit pas à son commerce et qui ne sortent pas de chez elles, celles qui rentrent trop tard du travail.

\section{Séquence 12.}

« Une femme dans la cité ", Maurice Dugowson, Les femmes... aussi, 1ère chaîne, 9 octobre 1972

Image 37. Arpenter les cages d'escalier

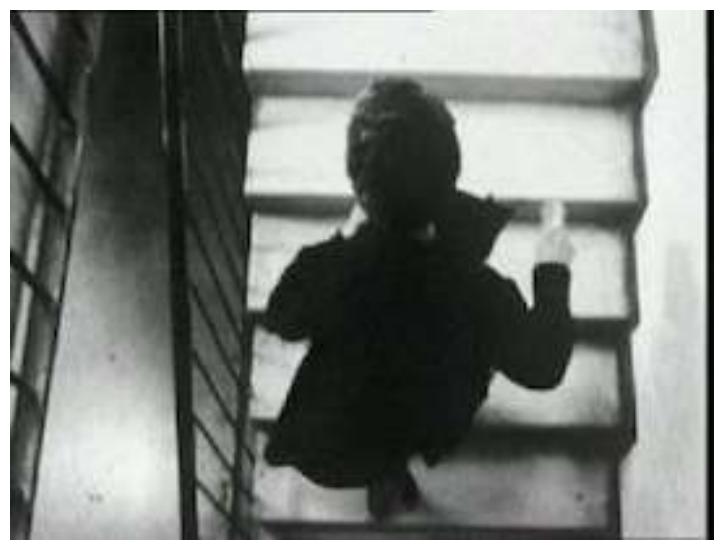

(CLes femmes aussi - 1972 
Image 38. Ouvrir une porte

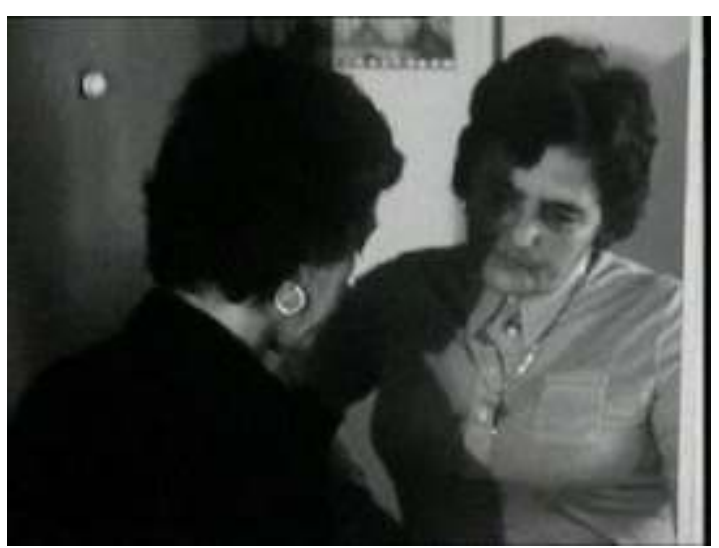

CLes femmes aussi - 1972

Image 39. Ouvrir une autre porte

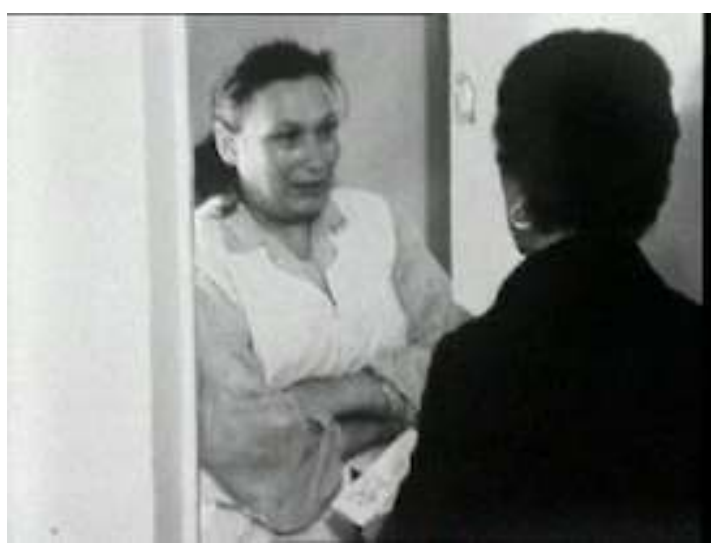

(c)Les femmes aussi - 1972

Image 40. Porte entrouverte

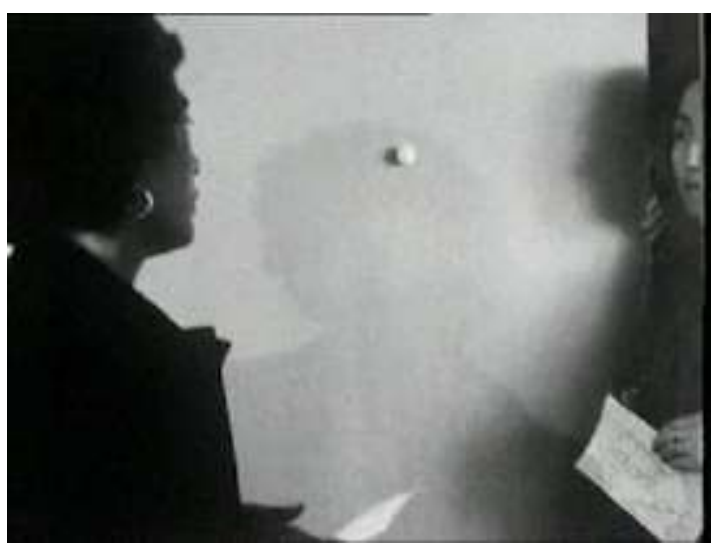

(c)Les femmes aussi - 1972

Elle fait donc du porte-à-porte l'après-midi, lorsque les femmes sont seules à la maison. Elle empreinte les escaliers sur la pointe des pieds pour ne pas faire de bruit. Sac à mains au bras, tracts en main, elle sonne une première fois. Personne ne vient ouvrir. 
Elle redescend d'un étage. Cette fois une femme ouvre, le tablier autour de la taille. Marie l'invite mercredi pour la réunion sur les femmes à la maison de quartier. La dame reste perplexe : "avec tout le travail que j'ai vous savez », alors Marie la gronde un peu « roooh mais vous ne sortez jamais quand-même ! ». Elle reprend son circuit, la caméra suit ces escaliers monotones et sonores, cette rambarde métallique qui n'en finit pas. Une autre porte s'ouvre. Puis une autre encore, mais la jeune femme prévient: son mari n'aime pas qu'elle aille là-bas. Marie termine par une dernière interaction. Elle donne à nouveau son tract « les femmes dans nos cités ». Encore une fois, l'habitante lui explique qu'elle est seule avec ses enfants et très prise par son travail. Les yeux fixés sur son interlocutrice, à quelques centimètres d'elle seulement, la tête penchée d'attention, Marie se veut compréhensive sans pour autant lâcher l'affaire : «jamais vous sortez, je sais que vous avez beaucoup de travail (...) je vous le laisse quand-même (...) si un jour vous pouvez venir avec Monique, je sais que vous ne sortez jamais, ça me ferait tellement plaisir de vous voir ».

Comme le spectateur a pu observer le travail domestique de Micheline, il peut suivre pendant trois minutes le long et laborieux travail de porte-à-porte de Marie. Il mesure son endurance à arpenter les cages d'escalier, à retenir l'attention de ces femmes au moins le temps d'un bref échange. À chaque fois, elle reste sur le palier, certaines femmes rabattent même la porte contre elle, osant à peine ouvrir. Elles restent "calfeutrées chez elles avec leurs problèmes" comme le regrette dans un autre reportage une habitante ${ }^{16}$. Ces portes maintiennent ostensiblement une frontière nette entre l'intérieur et l'extérieur, entre la vie domestique et la vie publique. Ce sont ces portes que voudrait faire ouvrir Marie, faire que les problèmes privés des femmes exprimés à demi-mot à l'occasion du porte-à-porte - deviennent publics, l'objet de discussion entre femmes: la conciliation de la vie familiale et du travail salarié, les interdictions imposées par les hommes, la solitude, la réclusion domestique. Sans doute, Marie fait-elle bien avant l'heure de l'empowerment comme on dirait aujourd'hui. Si les conseillères ménagères dans les centres sociaux accompagnent les femmes dans leur rôle de mère, d'épouse et de ménagère, Marie vise pour elles un devenir plus émancipé des rôles traditionnels. Par exemple, elle évoque à demi-mot la contraception avec un client: « et votre dame, elle va bien ? Vous n'avez plus de bébé ». L'homme : " oui, ça y est ». Et Marie de répondre, satisfaite : « oui, ça suffit maintenant ».

Après la séquence $d u$ porte-à-porte, succèdent une scène de réunion puis une assemblée générale.

Séquence 13.

"Une femme dans la cité ", Maurice Dugowson, Les femmes... aussi, 1ère chaîne, 9 octobre 1970 
Image 41. Assemblée générale

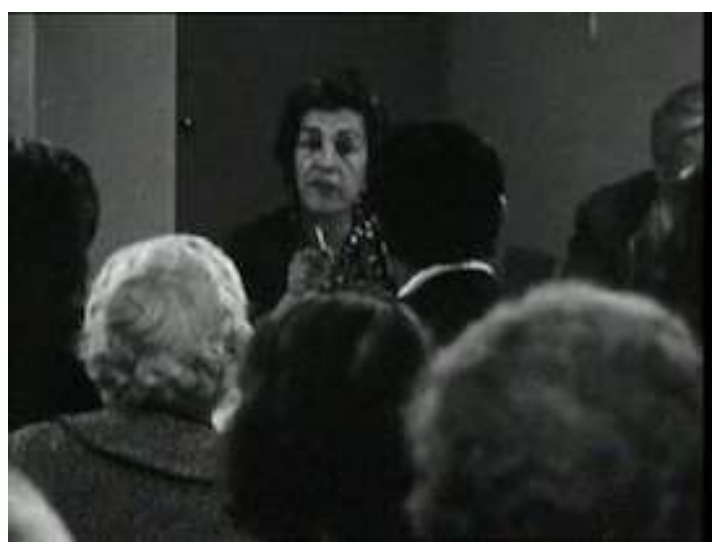

CLes femmes aussi - 1972

Image 42. Scène de réunion

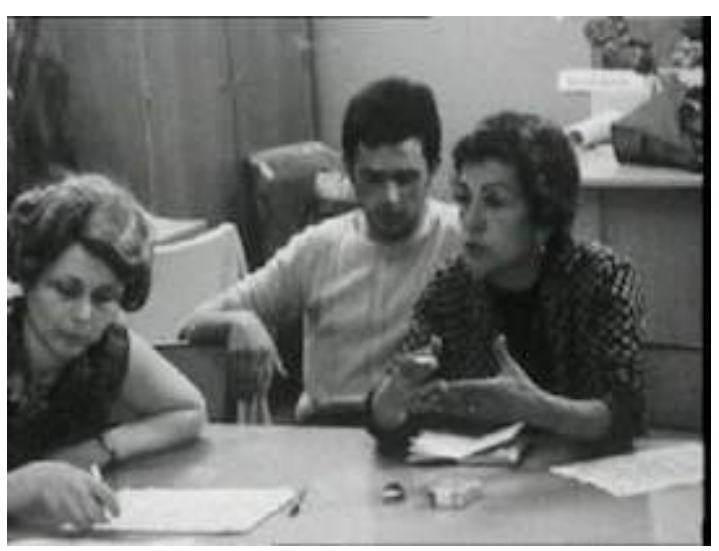

CLes femmes aussi- 1972

Le montage traduit la complémentarité des actions. Le métier de femmes en HLM a un accent de travail social communautaire, autrement dit un travail d'organisation et de renforcement de la communauté de quartier (Alinsky, 1971). Celle-ci commence à prendre forme dans cette bien nommée maison de quartier. À l'instar des organisateurs communautaires, Marie sollicite les femmes pour qu'elles participent et rejoignent cet "endroit où on peut se réunir pour découvrir certains problèmes ensemble ", parce qu' « à tous les étages, il y a des problèmes ». Les problèmes font lien dans la cité. Parmi ceux qui sont égrainés pendant les réunions : la drogue, le bus, les parkings, les travaux $\mathrm{du}$ stade, les expulsions locatives. Des habitants se rassemblent et cherchent collectivement des réponses : la maison et le journal de quartier, la fête de l'été, le comité des jeunes, une délégation pour protester contre le bailleur social.

« Une femme dans la cité ", Maurice Dugowson, Les femmes... aussi, 1ère chaîne, 9 octobre 1970 
Image 43. Spectacle à la maison de quartier

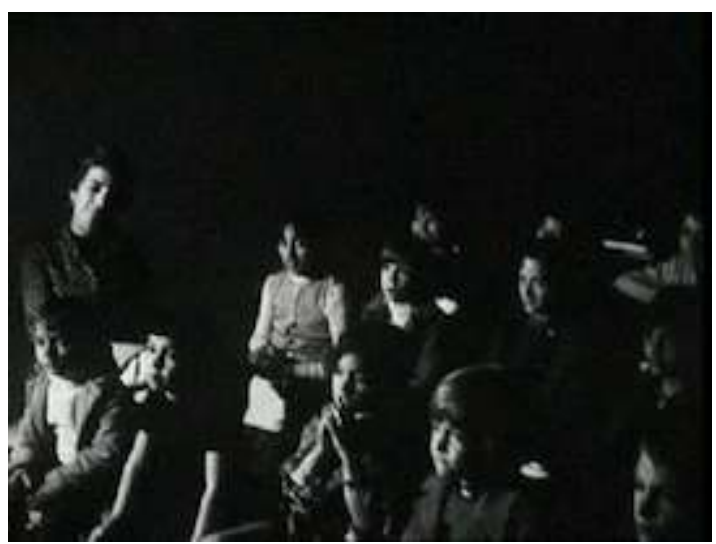

(c)Les femmes aussi- 1972

Depuis les prémices de l'aide sociale, la relation aux institutions incombe aux femmes comme l'a montré Richard Hoggart (1970). Marie écrivait dès l'âge de 8 ans des courriers administratifs pour ses parents arméniens, cela lui donne une expérience: « je fais attention [aux petits algériens qui traduisent pour leur mère], je suis passée par là ». Cette part du métier de femmes s'accroît et se complexifie dans les grands ensembles. Les guichets se font plus nombreux : il faut traiter avec les équipements sociaux, les offices HLM concernant le loyer et les charges, mais aussi la Caisse d'allocations familiales. Les conseillères ménagères interviennent également sur ce front. En assemblée, Marie informe les locataires sur leurs droits: l'un deux a peur d'être expulsé. À la tribune, elle répond en martelant chaque mot en hochant son stylo : « du moment que vous avez vos quittances, ce n'est pas possible qu'on vous donne congé ».

À Lyon aussi, dans le grand ensemble de la Duchère, une vie collective s'organise à travers l'Association Familiale et Culturelle de la Duchère. Ici, « les femmes jouent un rôle important ", si bien que c'est à deux d'entre elles que la télévision accorde en 1970 cinq minutes d'antenne. Filmées en plan fixe, assises derrière une table, le grand ensemble en fond, elles sont mises en position d'autorité.

Séquence 14.

« Les murs de la vie : le logement et l'urbanisme, Igor Barrère, L'Hexagone, 1ère chaîne, 7 avril 1970 


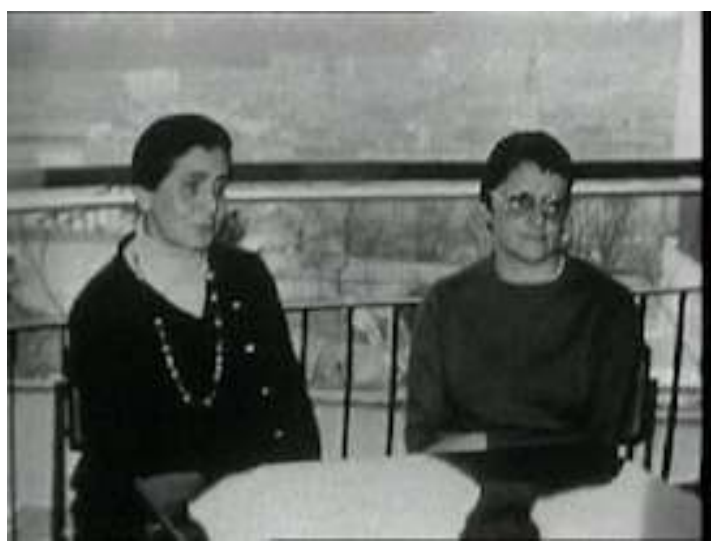

(C)Hexagone - 1970

Image 45. Programme d'activités du Centre Social du Plateau à la Duchère

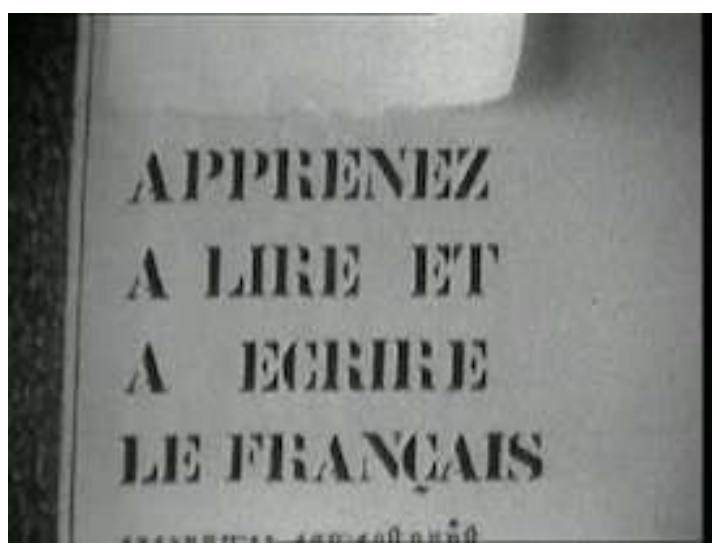

(C)Hexagone - 1970

Elles organisent «une vie collective et communautaire»: «on est arrivé dans un quartier tout neuf où personne ne se connaissait, il n'y avait pas encore d'habitudes prises, il y a eu un besoin de se serrer les coudes ». Cela passe par de nombreuses actions revendicatives à l'égard du bailleur, mais aussi par l'organisation de cours du soir pour les adultes et les jeunes travailleurs. Le métier de femmes en HLM renouvelle ainsi l'éducation populaire dans les années 1960. Les femmes s'auto-organisent et prennent directement en charge l'intervention sociale: lien entre habitants et institutions, hébergement d'urgence ou encore médiation familiale. Cette histoire est racontée dans le reportage : lorsqu'une jeune fille a fugué, elle est allée dormir chez la fille de l'une des militantes de l'association. Celle-ci a alors rétabli le lien avec les parents.

Ce sont donc des modalités nouvelles du travail social qui s'éprouvent à l'écran, et les femmes y ont un rôle clé. La télévision montre des initiatives fortes. Si certains commentaires insistent sur l'exemplarité de ce métier de femmes en HLM - « Marie, une femme parmi d'autres et pourtant pas tout à fait comme les autres » entend-on en préambule -, le sourire et le discours résolument optimistes des protagonistes finiraient par être contagieux... Des possibles s'ouvrent à l'orée des années 1968, qui seront marquées par les expérimentations collectives et les luttes urbaines. Des femmes 
éprouvent leur pouvoir d'intervention dans la cité sur ces terrains d'aventure que sont alors les grands ensembles. À travers les images de télévision qui s'en font l'écho, d'autres femmes s'éprouvent comme citoyenne.

\section{Conclusion. Dans l'ombre des archives}

51 Le métier de femmes en HLM prend consistance au croisement de l'histoire des grands ensembles, de l'émancipation des femmes et des premiers longs documentaires de la télévision des années 1960. Ce métier apparait sous de multiples facettes puisque les personnages filmés sont femme au foyer, conseillère ménagère, commerçante, animatrice-militante, ou encore infirmière à domicile. Et bien souvent, tout ça à la fois.

Ce métier de femmes en HLM se construit en tension entre le champ militant et le champ professionnel. Il relève d'abord du militantisme. Les grands ensembles se font le creuset d'un profond renouveau du travail social vers l'auto-organisation. Des femmes prennent en charge par elles-mêmes les problèmes de la cité. Un travail de nature politique et ancré dans la quotidienneté, à l'opposé du cadre professionnel assistanciel et spécialisé en guichets.

53 Une compétence crève l'écran : l'attention des femmes, comme pour déjouer l'image d'une femme indifférente, ou en dépression dans son HLM. Si l'attention est domestique, tournée vers la famille, elle est aussi publique, tournée vers le quartier. Cette double attention est largement soutenue par les éléments de confort du logement, les équipements collectifs, la diffusion de nouveaux savoirs. Arts, langues, puériculture, aménagement d'un appartement, atelier beauté, excursions en car : un ensemble d'activités surgissent si bien que de nouvelles subjectivités et compétences s'éprouvent.

Affleurent alors à l'écran les effets de l'urbanisation sur l'émancipation des femmes de milieu populaire. En premier lieu, si le métier de femmes en HLM ne libère pas celles-ci de la réclusion domestique, l'accès au confort diminue la pénibilité du travail ménager. Les situations observées dans le corpus sont sans commune mesure avec celles que vivent les habitantes des logements insalubres et des bidonvilles, soumises à la corvée d'eau plusieurs fois par jour par exemple.

Ensuite, grâce à d'autres femmes militantes, la maison de quartier et l'amicale des locataires engagent des actions revendicatives (coût du loyer, charges, misère économique, expulsion), tandis que le «club mamans » fait sortir les femmes de leur logis, les rassemble pour rompre l'isolement de celles qui ne retrouvent pas dans la cité l'animation urbaine des quartiers anciens.

56 Plus formellement, le grand ensemble est un terrain d'emplois féminins. Les archives nous font découvrir la " conseillère ménagère ", un métier de femmes au sens commun du terme, c'est-à-dire une activité exercée exclusivement par des femmes dans des espaces spécifiques: les équipements sociaux. Celle-ci fait la promotion de nouvelles manières d'habiter sans bouleverser le rôle traditionnel de la femme au foyer. À l'inverse, les militantes font du bruit dans une visée émancipatrice. Au fil du temps, certaines sont promues salariées des équipements et doivent alors concilier militantisme et cadre professionnel. Dans les années 1990, le dispositif d'emploi des femmes-relais formalise ce métier pour retisser le lien entre habitants et institutions. 
Des femmes sont alors recrutées pour leurs relations sociales dans la cité, une manière de faire de la connaissance des banlieues une profession (Profession Banlieue, 1997).

Enfin, on peut penser que la politique d'accès à la culture de l'après-guerre produit ses premiers effets au cœur des HLM. Les femmes disent par exemple "apprendre beaucoup » en regardant la télévision, elles entendent d'autres femmes parler. Et à leur tour, elles s'adressent aux téléspectatrices, à l'instar de Micheline qui nous met en garde sur la condition de mère au foyer. C'est aussi en suivant la scolarité de leurs enfants, qu'elles découvrent ce que peut être l'école et critiquent l'enseignement ménager qu'elles ont reçu. L'annonce d'une rupture émancipatrice? Probablement pas, mais une série de petits déplacements qui leur font regarder les choses autrement.

Le métier de femmes en HLM s'exprime sur l'écran dans un jeu de visibilité et d'invisibilité. Le travail ménager apparait dans sa complexité tout autant technique qu'humaine - le sens de la responsabilité, la dimension affective. Nous retenons aussi du corpus les images du corps des femmes au travail. Corps discipliné par les arts ménagers, corps vulnérable dans ces cités de béton, des corps pourtant vifs : ils arpentent le grand ensemble à grandes enjambées, ils frottent avec vigueur le petit linge, ils sont capables de tant d'émotions. Pour la première fois, ces corps vifs sont montrés à l'écran.

Pour autant, un point aveugle des archives est particulièrement marquant : les femmes immigrées. Quelques reportages font exception. L'un d'eux donne assez longuement la parole à une femme algérienne : comme les mères au foyer françaises, elle raconte le budget familial, la scolarité de ses enfants, les courses au marché pour acheter «le persil algérien »- la coriandre ${ }^{17}$. Cette absence est troublante. Au tournant des années 1970, ces ménages accèdent bon an mal an aux cités de transit et aux HLM (BlancChaléard, 2016). Les producteurs de télévision pensent-ils, comme les autorités de l'État, que les étrangers ne sont que de passage ? À quoi bon alors les entendre sur leur logement? Trente ans plus tard, ce sont ces femmes et leurs filles qui sont dans l'œil des producteurs de documentaires sur les cités HLM, tantôt montrées comme femmes actives et médiatrices (les précieuses femmes-relais), tantôt comme victimes. D'invisibles, elles finissent par ne plus pouvoir échapper à la médiatisation. Depuis les années 1960, ce jeu du visible/invisible leur échappe.

Ces images du travail des femmes participent d'une mémoire collective des femmes. Derrière la figure des ménagères filmées dans leur quartier alors flambant neuf, nous voyons se profiler des figures plus contemporaines, des animatrices, des femmes ordinaires qui vivent et travaillent dans les cités HLM. Ces archives nous ont montré des pratiques qui s'étaient forgées au cœur des années 1960. C'est déjà une manière de voir les femmes d'aujourd'hui sous un autre jour.

\section{BIBLIOGRAPHIE}

Alinsky S. (1976) [1971], Manuel de l'animateur social, Paris, Points. 
Bernège P. (1928), De la méthode ménagère, Orléans, Tessier.

Bessin M. et Gaudart C. (2009), « Les temps sexués de l'activité : la temporalité au principe du genre? ?, Temporalités, $\mathrm{n}^{\circ} 9$.

Blanc-Chaléard M-C (2016), En finir avec les bidonvilles. Immigration et politique du logement dans la France des Trente Glorieuses, Paris, Publications de la Sorbonne.

Brayet A. (2010), «L'image et la fée du logis : former les femmes à devenir de bonnes ménagères ", in F. Laot (dir.), L'image dans l'histoire de la formation des adultes, Paris, L'Harmattan, p. 49-68.

Canteux C. (2014), Filmer les grands ensembles, Créaphis Éditions.

Chabaud-Rychter D., Fougeyrollas-Schwebel D. et Sonthonnax F. (1985), Espace et temps du travail domestique, Paris, Librairie des Méridiens.

Chombart de lauwe M-J. (1964), « Image de la situation de la femme dans la société urbaine française ", in Chombart de Lauwe P.-H., Image de la femme dans la société, Paris, Éditions Ouvrières, p. 41-96.

Clarke J. (2005), « L'organisation ménagère comme pédagogie. Paulette Bernège et la formation d'une nouvelle classe moyenne dans les années 1930 et 1940 », Travail, genre et sociétés, n 13 , p. 139-157.

Cohen E. et Lévy M-F. (dir.), 2007, La télévision des Trente Glorieuses. Culture et politique, Paris, CNRS Editions.

Denèfle S. (dir.), 2004, Femmes et ville, Tours, Presses universitaires François-Rabelais.

Faure D. (1992), « La conseillère ménagère à E.D.F », Bulletin d'histoire de l'électricité, n 19-20, p. 199-213.

Girard A. (1958), « Le budget-temps de la femme mariée dans les agglomérations urbaines », Population, $\mathrm{n}^{\circ}$ 4, p. 591-618.

Grégoire M. (1964), Le métier de femmes, Paris, Plon.

Hoggart R. (1970), La culture du pauvre, Paris, Éditions de Minuit.

INSEE (1969), Résultats préliminaires du recensement de 1968 : démographie générale, population active, ménages, logements, Paris, Insee.

Laot F. F. (2014), Un film comme source pour l'histoire de la formation des adultes hommes... et femmes. «Retour à l'école? », (Nancy, 1966), Nancy, PUN Éditions universitaires de Lorraine.

Lebeaume J. (2014), L'enseignement ménager en France. Sciences et techniques au féminin 1880-1980, Rennes, PUR.

Lévy M-F. (1995), « Famille et télévision 1950-1986 », Réseaux, n 72-73, p. 177-193.

Lévy M-F. (2007), « Télévision, public et citoyenneté », in E. Cohen et M-F Lévy (dir.), La télévision des trente glorieuses, Paris, CNRS éditions.

Martin M. (1987), « Ménagère : une profession ? Les dilemmes de l'entre-deux guerres », Le mouvement social, $\mathrm{n}^{\circ} 140$, p. 89-106.

Overney L. (2014), « Par-delà "la participation des habitants" : pour une ethnographie de la petite politique. Le cas d'un collectif d'habitants de la Duchère », in : M. Carrel et C. Neveu (dir.), Citpoennetés ordianires : pour une approche renouvellée des pratiques citoyennes, Paris, Khartala, p. 131-166. 
Peneff J. (2009), Le goût de l'observation, Paris, La Découverte.

Perrot M. (1980), « La ménagère dans l'espace parisien au XIXe siècle », Les annales de la recherche urbaine, $\mathrm{n}^{\circ} 9$, p. 3-22.

Profession Banlieue (1997), Professionnaliser la médiation sociale. Pour un statut des femmes-relais, Saint-Denis, Profession Banlieue.

Rancière J. (1993), « L'histoire « des » femmes : entre subjectivation et représentation » (note critique), Annales. Économies, Sociétés, Civilisations, n 4, p. 1011-1018.

Rochefort C. (1961), Les petits enfants du siècle, Paris, Grasset.

Science et vie, (1959), $\mathrm{n}^{\circ} 504$.

Sohn A-M. (1997), « Pour une histoire de la société au regard des médias », Revue d'histoire moderne et contemporaine, $\mathrm{n}^{\circ} 2$, p. 287-306.

Tellier T. (2007), Le temps des HLM, 1945-1975, Paris, Autrement.

Tellier T. (2008), « À quoi rêvent-elles ? La part des femmes dans la vie social des nouveaux ensembles urbains. Années 1960-1970 », in S. Denèfle (dir.), Utopies féministes et expérimentations urbaines, Rennes, PUR, p. 35-46.

Zancarini-Fournel M. et Artières P. (dir.), 2008, 68, une histoire collective [1962-1981], Paris, La Découverte.

\section{ANNEXES}

\section{Corpus étudié}

« La femme chez elle », Le magazine féminin, 27 février 1959, 1ère chaîne, 18'12.

Le magazine féminin, 5 mai 1960, 1ère chaîne, 30 mn.

«Quarante mille voisins », Pierre Tchernia, Jacques Krier, Cinq colonnes à la Une, 2 décembre 1960, 1ère chaîne, 14'21.

«Visite d'un appartement témoin HLM à Bron Parilly », Actualités Rhône Alpes, 2 décembre 1960, 2’21.

« Les arts ménagers au service du foyer », Des richesses et des hommes, 27 novembre 1961, 1ère chaîne, 24'40.

« Le bras favé », Jean-Christophe Averty, Jean-Claude Bringuier, Cinq colonnes à la Une, 1er juin 1962,1ère chaîne, 15’55.

" Aide à la détresse », Jean-Claude Lagneau, L'avenir est à vous, 2 mars 1964, 1ère chaîne, $24^{\prime} 48$.

"Conseillère ménagère », Anne-Marie Ullmann, L'avenir est à vous, 16 mars 1964, 1ère chaîne, 9'28.

"Jeunes dans une ville neuve », Jean-Pierre Chartier, L'avenir est à vous, 10 septembre 1964, 1ère chaîne, 23’59. 
« Ils ont trouvé un appartement », François Gir, Cinq colonnes à la une, 5 février 1965, 1ère chaîne, 11'29.

« Micheline, 6 enfants, allée des Jonquilles », Claude Goretta, Les femmes... aussi, 24 avril 1967, 1ère chaîne, 55’07.

« Le jasmin perdu », Serge Moati, Les femmes aussi, 7 janvier 1970, 1ère chaîne, 52'.

« Le prix du deuxième », Jacques Krier, Les Femmes... aussi, 11 février 1970, 1ère chaîne, $46^{\prime} 40$.

«Les murs de la vie : le logement et l'urbanisme, Igor Barrère, trois reportages :

« Famille HLM », « La Duchère ", « Saint-Chamond », L'Hexagone, 7 avril 1970, 1ère

chaîne, 1 h10.

«La vie d'une femme dans le Val d'Yerres », Aujourd'hui Madame, 8 juillet 1970, 2ème

chaîne.

« Une famille budget 800 frcs Biarritz », JT 20h, 25 août 1970, 5’15.

«Sarcelles », Aujourd'hui Madame, 25 février 1971, 1ère chaîne, 1 h01.

«Les femmes et la piscine ", JT de 13h, 12 mai 1971, 1ère chaîne.

« Hebdo Femmes », JT 13H, 23 juin 1971, 11'28.

« Le pain amer : l'escalier de Nanterre », Jacqueline Collins, Jacques Darribehaude, JeanVincent Fournis, Le troisième cil, 28 avril 1972, 2ème chaîne, 25'25.

« Ils ont fait cette ville », Jean-Pierre Chartier, Janick Arbois, Signes des temps, 24 juillet 1972, 2ème chaîne, 58'35.

« Une femme dans la cité », Maurice Dugowson, Les femmes... aussi, 9 octobre 1972, 1ère chaîne, 54'15.

«L'enfer du décor », Jacques Fremontier, Victoria Llanso, La vie ensemble, 8 juillet 1973, 2e chaîne, 1 h04.

\section{NOTES}

1. "Quarante mille voisins », Cinq colonnes à la Une, 2 décembre 1960.

2. Au recensement de la population de 1954, en France, $90 \%$ des logements n'ont ni douche ni baignoire, $73 \%$ ne disposent pas de W.C intérieurs, et $42 \%$ n'ont pas de l'eau courante (Insee, 1969).

3. La « sarcellite » trouve son étymologie dans cette ville emblématique des grands ensembles de la région parisienne : Sarcelles. Il apparaît pour la première fois en 1963 dans France soir (Tellier, 2007, 84). La formule relie les problèmes matériels ou fonctionnels de ce type d'habitat à un ensemble de "pathologies" sociales, médicales et psychiatriques. Le magazine Science et vie publie ainsi les résultats d'enquêtes sur " la maladie des HLM" dans lesquels psychiatres et sociologues s'alarment de la «névrose des banlieues" (Science et vie, 1959, 32-33). Les femmes sont la principale cible de cette littérature pseudo-scientifique, comme pour mieux esquiver d'autres problèmes : la pauvreté des familles logées en HLM, les inégalités dans les rapports entre les sexes, la société corsetée de l'avant 68, etc. Pour un autre aperçu de la vie des femmes et des jeunes filles troublées par les grands ensembles, on préférera la lecture du roman de Christiane Rochefort Les petits enfants du siècle (1961). 
4. Les constructions s'accélèrent à la fin des années 1950 pour faire face à la crise du logement, notamment à travers les Zones à Urbaniser en Priorité (ZUP). De nouveaux quartiers sortent de terre, des grands ensembles de plus de mille logements qui portent la marque de l'architecture moderne. La construction atteint son pic en 1973 avec 556000 logements construits. La circulaire Guichard du 21 mars 1973 finit par interdire la construction de grands ensembles.

5. Ce pli de l'enquête approfondie s'expérimente dès les années 1950 avec des émissions qui cherchent à initier les français aux problèmes de la cité. C'est le cas de la magnifique série À la découverte des Français, entre autres les documentaires de l'anthropologue Paul-Henry Chombart de Lauwe.

6. Les femmes du documentaire de Jacques Krier «Le prix du deuxième », Les Femmes... aussi, en 1970 n'arrêtent pas de compter : salaire brut, reste à vivre, dépenses pour la nourrice, transport, allocation logement, faible intérêt financier à poursuivre une activité professionnelle après un deuxième enfant.

7. Ils apparaissent dans les années 1950 sur le modèle des magazines féminins de la presse écrite et de la radio.

8. Paulette Bernège formalise en France l'enseignement ménager dans les années 1920 influencée par la domestic science anglo-saxonne de C. Frederick (Bernège, 1928). Les compétences de la ménagère sont élevées au rang de techniques scientifiques. Ainsi, elle devient une véritable gestionnaire, instruite aux techniques de rationalisation du travail domestique. Sur les débats qui entourent dans l'entre-deux guerres la reconnaissance du travail ménager comme un métier voir Martin (1987). Pour une histoire de l'enseignement ménager comme une voie de professionnalisation spécifique des femmes, voir Lebeaume (2014).

9. Le magazine féminin, émission du 5 mai 1960.

10. « Ils ont trouvé un appartement », cinq colonnes à la une, 5 février 1965.

11. Ajoutons que les connaissances psychologiques font leur entrée dans l'espace public. Dès 1950, Françoise Dolto anime des émissions sur l'éducation sexuelle des enfants sur la RTF. Puis, en 1968/1969, sur Europe $n^{\circ} 1$, elle répond en direct aux questions des auditeurs.

12. Les habitantes des grands ensembles n'ont d'ailleurs pas répondu avec enthousiasme aux cours ménagers qu'on leur proposait. Thibault Tellier montre comment à Sarcelles les centres étaient peu fréquentés et inadaptés aux vraies demandes (2008).

13. «Une femme dans la cité », Les femmes... aussi, 1ère chaîne, 9 octobre 1972.

14. "Jeunes dans une ville neuve ", L'avenir est à vous, 10 septembre 1964.

15. « Ils ont fait cette ville », Signes des temps, 24 juillet 1972.

16. «La vie d'une femme dans le Val d'Yerres », Aujourd'hui Madame, 8 juillet 1970.

17. « Le pain amer : l'escalier de Nanterre » Le troisième œil, 28 avril 1972.

\section{RÉSUMÉS}

L'article décrit le travail des femmes dans les grands ensembles en s'appuyant sur des sources encore peu exploitées - les nombreuses archives télévisuelles des années 1960. Quelles pratiques et quelles attitudes, quels savoirs et savoirs faire sont alors mis en vue par les documentaires de la télévision? L'article se concentre particulièrement sur deux femmes : l'une se consacre sans relâche au travail domestique tandis que l'autre s'investit dans l'action sociale communautaire pour le quartier. Ce métier de femmes en HLM prend consistance au croisement de la politique de 
construction de l'après-guerre, de l'émancipation des femmes et des expériences documentaires de la télévision des années 1960. Il se construit en tension entre le champ militant et le champ professionnel. Il apparaît sous de multiples facettes puisque les personnages filmés sont femme au foyer, conseillère ménagère, commerçante, animatrice-militante, ou encore infirmière à domicile. Et bien souvent, tout ça à la fois. Les effets de l'urbanisation sur l'émancipation des femmes de milieu populaire affleurent alors à l'écran.

Based on the 60's television archives, sources that are by and large ignored in research, this paper describes the women's work in the social housing, in the high-rise estates. What kind of activities and attitudes, what kind of knowledge and expertise are shown on TV? This paper is centered on two women. One does household chores all day long whereas the other is involved in the community social work. The women's work is analysed by crossing the Reconstruction politics into the postwar period, the empowerment of women and the documentary films from the Sixties. It emerges between the professional sector and the militancy. It is shown from various angles : in these films, the women are : a housewife, a specialist of Home Economics, a shopekeeper, a community organizer, a visiting nurse. Often, they are all of these. The empowerment of women from the working-class comes up on the screen.

\section{INDEX}

Mots-clés : femmes, travail domestique, H.L.M, animation sociale, archives télévisuelles Keywords : women, housework, social housing, community social work, television archives

\section{AUTEUR \\ LAETITIA OVERNEY}

Laetitia Overney est sociologue, maîtresse de conférences à l'Ecole Nationale Supérieure d'Architecture de Paris-Belleville et chercheure à l'Ipraus-Umr AUSser. Son travail porte principalement sur les formes d'expressions publiques dans les quartiers populaires. Elle a longuement enquêté dans des quartiers en rénovation urbaine en suivant les prises de parole des habitants. Elle mène actuellement une recherche socio-historique sur le rôle des femmes les dans grands ensembles dans les années 1960 et 1970. 